\title{
An alternative approach to the ergodic theory of measured foliations on surfaces
}

\author{
MARY REES \\ School of Mathematics, University of Minnesota, Minneapolis, MN 55455, USA
}

(Received 1 June 1981)

\begin{abstract}
We consider measured foliations on surfaces, and interval exchanges. We give alternative proofs of the following theorems first proved by Masur and (independently) Veech. The action of the diffeomorphism group of the surface on the projective space of measured foliations (with respect to a natural 'Lebesgue' measure) is ergodic. Almost all measured foliations are uniquely ergodic. Almost all interval exchanges (again, with respect to a natural 'Lebesgue' measure) are uniquely ergodic.
\end{abstract}

\section{Introduction}

In this paper we give alternative proofs to two theorems first proved by Masur [5] about measured foliations on surfaces. Recall that a measured foliation on a surface is defined [2] as being a $C^{\infty}$ foliation except at finitely many points, in neighbourhoods of which leaves are images of the lines $\left\{\operatorname{Im} Z^{P / 2}=\right.$ const $\}$ ( $p \geq 3$, or $p \geq 4$ if the point is on the boundary of the surface) for a chart in the complex plane containing 0 . Also, the foliation is endowed with a finite invariant transverse measure $C^{\infty}$-equivalent to Lebesgue. The theorems are:

THEOREM 1. Let $\operatorname{Diff}_{0}\left(M_{g, b}\right)$ be the modular group of the oriented surface $M_{g, b}$ with genus $g$ and $b$ boundary components. Let $\mathcal{M F}\left(M_{g, b}\right)$ denote the space of measured foliations on $M_{g, b}$, and $\mathscr{P} \mathscr{M F}\left(M_{g, b}\right)$ the projective space. Then $\operatorname{Diff}_{0}\left(M_{g, b}\right)$ acts ergodically on $\mathscr{P} \mathscr{M} \mathscr{F}\left(M_{\mathrm{g}, b}\right)$ with respect to Lebesgue measure.

THEOREM 2. Lebesgue-almost-all foliations in $\mathscr{M} \mathscr{F}\left(M_{g, b}\right)$ are uniquely ergodic (up to multiplying the measure by a scalar).

We explain the terminology in $\S 1$. Masur actually proved a stronger result than theorem 1 - that the action on the square of $\mathscr{P} \mathscr{M} \mathscr{F}\left(M_{g, b}\right)$ is ergodic. The methods of the present paper give conservativity of the action on the square. We recall that closely related results were proved by Masur [5], and by Veech [7], for interval exchanges. We include a proof of this, although the aim is rather to give a different viewpoint on theorems 1,2 :

THEOREM 3. Lebesgue-almost-all irreducible interval exchanges are uniquely ergodic.

An irreducible permutation $\sigma$ of $\{1, \ldots, n\}$ is one such that

$$
\sigma\{1, \ldots, r\} \neq\{1, \ldots, r\} \text { for any } r<n .
$$


For any $\lambda=\left(\lambda_{1}, \ldots, \lambda_{n}\right), \lambda_{i} \geq 0$, if $\beta_{i}=\sum_{j \leq i} \lambda_{j}$, with $\beta_{n}>0$, define $f_{\lambda, \sigma}:\left[0, \beta_{n}\right) \rightarrow\left[0, \beta_{n}\right)$ by:

$$
f_{\lambda, \sigma}\left(x+\beta_{i-1}\right)=x+\sum_{\sigma(j) \leq \sigma(i)-1} \lambda_{j}, \quad 0 \leq x<\lambda_{i} .
$$

'Lebesgue-almost-all' in the statement of theorem 3 then means: for Lebesguealmost-all $\lambda$, for a fixed $\sigma$.

The proofs of theorems 1,2 are inextricably linked. The same sort of situation occurs in [5], [7]. Veech uses the conservativity of $\left(\mathscr{P} \mathcal{M} \mathscr{F}, \operatorname{Diff}_{0}\left(M_{g, b}\right)\right.$, Lebesgue) to prove unique ergodicity (although the language is completely different) and Masur uses conservativity of the square. In the case of measured foliations of the torus, theorem 1 becomes the classical theorem of the ergodicity of $(\mathbb{R} \cup\{\infty\}, \operatorname{SL}(2, \mathbb{Z})$, Lebesgue) where

$$
\left(\begin{array}{ll}
a & b \\
c & d
\end{array}\right) \cdot x=\frac{a x+b}{c x+b}, \quad x \in \mathbb{R} \cup\{\infty\}, \quad\left(\begin{array}{ll}
a & b \\
c & d
\end{array}\right) \in \operatorname{SL}(2, \mathbb{Z}) .
$$

One classical proof of this involves showing that the action of $\operatorname{SL}(2, \mathbb{Z})$ on $\{z \in \mathbb{C}: \operatorname{Im} z>0\}$ given by

$$
\left(\begin{array}{ll}
a & b \\
c & d
\end{array}\right) \cdot z=\frac{a z+b}{c z+d}
$$

has a finite volume quotient. Veech and Masur obtain the needed conservativity results by proving an analogue of this. (Of course, this is a gross over-simplification.) In the present paper, we imitate a different proof of the ergodicity of $(\mathbb{R} \cup\{\infty\}, \operatorname{SL}(2, \mathbb{Z})$, Lebesgue) exposed in [6]:

Let $E \subseteq \mathbb{R} \cup\{\infty\}$ be a set of positive measure. After acting by

$$
\left\{\left(\begin{array}{ll}
1 & n \\
0 & 1
\end{array}\right): n \in \mathbb{Z}\right\}
$$

we may assume that $E \subseteq(0,1)$. Let $x$ be a density point of $E$, and irrational. For infinitely many $q>0$, we have $p>0$ with

$$
|p / q-x|<1 / q^{2}
$$

There are $r, s>0$ with $s<q$ and $r q-s p=1$. So the derivative of $\left(\begin{array}{ll}r & p \\ s & q\end{array}\right)$ on $(0,1)$ is $O\left(1 / q^{2}\right)$, and $\left(\begin{array}{ll}r & p \\ s & q\end{array}\right)$ maps $(0,1)$ to an interval of width $>1 / 2 q^{2}$ in $\left(x-2 / q^{2}, x+2 / q^{2}\right)$. Since $x$ is a density point, the inverse image of $\left(x-2 / q^{2}, x+2 / q^{2}\right) \cap E$ under $\left(\begin{array}{cc}r & p \\ s & q\end{array}\right)$ contains arbitrarily much of the measure of $(0,1)$ as $q \rightarrow \infty$. Translation of $(0,1)$ by

$$
\left\{\left(\begin{array}{ll}
1 & n \\
0 & 1
\end{array}\right) ; n \in \mathbb{Z}\right\}
$$

then gives that the orbit of $E$ is almost all $\mathbb{R} \cup\{\infty\}$.

Note that a generalization of this method might (and does) yield the following: if $E \subseteq \mathscr{P} M \mathscr{F}$ has positive measure, then there exists a sequence $\left\{\phi_{n}\right\} \subseteq$ Diff $_{0}$ such that

$$
\text { measure }\left(\mathscr{P} \mathcal{M F}-\phi_{n}^{-1} E\right) \rightarrow 0
$$


One might also suspect that, as for actions of groups of hyperbolic isometries, if the Radon-Nikodym derivative of $\phi \in \operatorname{Diff}_{0}\left(M_{g, b}\right)$ on a set $U$ is uniformly small, then not only measure $(\phi U)$, but also diameter $(\phi U)$ is small. This is not at all true. It is, however, true in a 'probabilistic' sense (proposition (3.1)). It is, in fact, here that the problem of unique ergodicity arises.

One possible way of explaining why the ergodic theory of $\mathscr{P} \mathcal{M F}\left(M_{g, b}\right)$ is less straightforward in general than in the classical case of the torus, is that the natural analogue of a conformal metric for $\mathscr{P} \mathscr{M} \mathscr{F}$ is not a metric at all, but the intersection number. We use the intersection number in the present paper. We include $(\S 1)$ some basic facts about intersection numbers which do not appear in [2] (although known). $\S 1$ also contains other basic facts about measured foliations.

$\S 2$ concerns multiple loops on surfaces - the natural generalization of rationals in $\mathbb{R} \cup\{\infty\}$. The idea is to prove that a positive measure set of measured foliations can be 'well approximated' by 'prime' multiple loops. (We do not try for a full measure set at first.) This is done essentially by generalizing the result that the number of coprime pairs $(p, q)$ with

$$
a N \leq|p|+|q|<b N \quad(0<a<b)
$$

is $O\left(N^{2}\right)$. A similar result is needed for theorem 3 . The divergence from straight imitation of the classical case essentially comes in $\S 3$, where we use analysis (mostly a simple application of the Birkhoff ergodic theorem) to prove the existence of 'density points' for any subset of positive measure of a certain subset of $\mathscr{P} \mathscr{M} \mathscr{F}$ with respect to a certain cover of this subset.

My fundamental debt to [2] will be clear. I should like to thank, in particular, A. Fathi and F. Laudenbach for their help, encouragement and additional information.

\section{Basic facts about measured foliations}

We consider the space of measured foliations

$$
\mathscr{M F}\left(M_{g, b}\right)=M \mathscr{F}
$$

on the oriented surface $M_{g, b}$ of genus $g$ with $b$ boundary components. The basic reference is [2].

An intersection number $i(\alpha, \mathscr{F})$ is defined for any simple (that is, non-self-intersecting) loop $\alpha$ on $M_{g, b}$, and $\mathscr{F} \in \mathcal{M} \mathscr{F}$, as the minimal measure given by $\mathscr{F}$ to a loop in the isotopy class of $\alpha$. Recall that $\mathscr{F}_{1}, \mathscr{F}_{2}$ are considered to be equivalent if

$$
i\left(\alpha, \mathscr{F}_{1}\right)=i\left(\alpha, \mathscr{F}_{2}\right) \text { for all } \alpha .
$$

An $\mathbb{R}^{+}$-action is defined on $\mathscr{M F}$ by taking $\lambda \cdot \mathscr{F}$, for $\lambda \in \mathbb{R}^{+}$, to be the same foliation as $\mathscr{F}$, with all transverse measures multiplied by $\lambda$.

An action of the modular group $\operatorname{Diff}_{0}\left(M_{g, b}\right)$ is defined on $\mathscr{M} \mathscr{F}$ by

$$
[\phi] \cdot[(\mathscr{F}, \mu)]=\left[\left(\phi \mathscr{F}, \phi_{*} \mu\right)\right] \text {, }
$$

where [ ] denotes an equivalence class, and

for any transversal $I$.

$$
\phi_{*} \mu(I)=\mu\left(\phi^{-1} I\right)
$$


The piecewise GL $(\cdot, \mathbb{Z} / 4)$-structure of $\mathcal{M F}$

The following theorem is well known. Most comes directly from [2]. $\mathbb{Z} / 4$ denotes the group of rationals with denominator 4 . We shall elaborate some details.

(1.1) THEOREM. $\mathscr{M F}\left(M_{g, b}\right)$ has a structure of a piecewise $\operatorname{GL}\left(\mathbb{R}^{6 g-6+2 b}, \mathbb{Z} / 4\right)$ manifold. The charts can be taken as $\mathbb{R}^{+}$-invariant, the $\mathbb{R}^{+}$-action translating to scalar multiplication in $\mathbb{R}^{6 \mathrm{~g}-6+2 b}$. The $\operatorname{Diff}_{0}\left(M_{\mathrm{g}, b}\right)$-action is piecewise $\mathrm{GL}\left(\mathbb{R}^{6 \mathrm{~g}-6+2 b}, \mathbb{Z} / 4\right)$. There is a Lebesgue measure $\lambda$ on $\mathcal{M F}$ such that the $\left|d \phi_{*} \lambda / d \lambda\right|$ are uniformly bounded for $\phi \in \operatorname{Diff}_{0}\left(M_{\mathrm{g}, b}\right)$.

For the last part of the theorem we simply take a disjoint set of charts, and the Lebesgue measures from these. The boundedness of the $\left|d \phi_{*} \lambda / d \lambda\right|$ follows from the fact that a matrix $A$ with both $A, A^{-1}$ in GL $(n, \mathbb{Z} / 4)$ has determinant between $1 / 4^{n}$ and $4^{n}$.

Note. By slightly more sophisticated methods, Masur actually finds an invariant measure on $\mathscr{M F}[5]$.

We should maybe elaborate on the choice of coordinates which makes the structure piecewise GL $(6 g-6+2 b, \mathbb{Z} / 4)$, not merely piecewise linear.

From [2] there are finitely many multiple loops

$$
\boldsymbol{\alpha}=\left(\alpha_{1}, \ldots, \boldsymbol{\alpha}_{3 g^{-3+b}}\right)
$$

with the $\alpha_{i}$ isotopically distinct disjoint simple loops, such that the sets $X_{\alpha}$ cover $\mathcal{M F}$, where

$$
X_{\boldsymbol{\alpha}}=\left\{\mathscr{F}: i\left(\alpha_{i}, \mathscr{F}\right)>0 \text { for all } j\right\}
$$

Write $m_{j}=i\left(\alpha_{j}, \mathscr{F}\right)$. The $m_{j}$ determine $\mathscr{F}$ restricted to each pair of pants into which $\alpha$ cuts $M_{g, b}$. An $\mathscr{F} \in X_{\alpha}$ looks like figure 1 in a pair of pants bordered by $\alpha_{i_{1}}, \alpha_{i_{2}}, \alpha_{i_{3}}$.
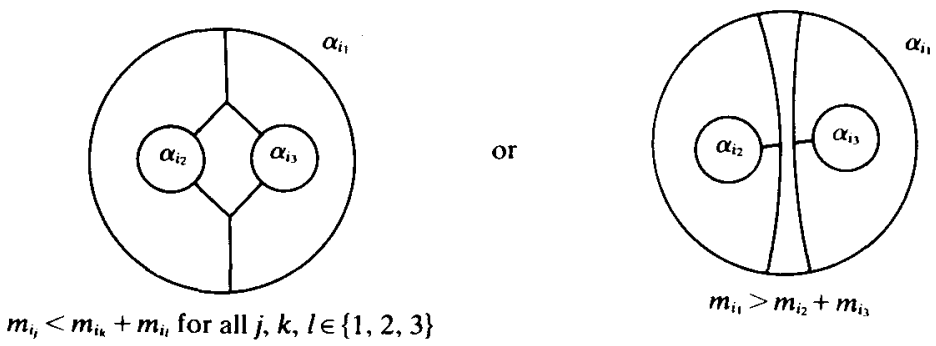

Figure 1

There are two other cases obtained from the latter by interchanging $\alpha_{i_{1}}, \alpha_{i_{2}}, \alpha_{i_{3}}$, and borderline cases such as $m_{i_{1}}=m_{i_{2}}+m_{i_{3}}$. (Pictures are given in [2, exp. 6].) For each $\alpha_{j}$, and each possible pair of configurations occurring for foliations in $X_{\alpha}$, in the pairs of pants bordering on $\alpha_{j}$ (it does not matter if there is only one pair of 
pants bordering on $\alpha_{j}$ ), choose a singularity and singular prong in each of the one or two pairs of pants such that the two singular prongs hit $\alpha_{j}$ on opposite sides of $\alpha_{j}$. Choose also a homotopy class of arc crossing $\alpha_{i}$ and joining the two singularities. (This still makes sense if the two singularities coincide.) Choose also an orientation on $\alpha_{j}$, and call one of the two singular prongs the 'first'. Given $\mathscr{F}$ in $X_{\alpha}$, let $p_{i}$ denote how far in measure the second singular prong has to be moved along $\alpha_{j}$-positively or negatively - to meet the first singular prong and form the chosen arc, up to homotopy. The coordinates $\left(m_{j}, p_{j}\right)_{i=1, \ldots, 3 g-3+b}$ completely determine $\mathscr{F} \in \boldsymbol{X}_{\mathbf{\alpha}}$. Let

$$
\Phi_{\alpha}: X_{\alpha} \rightarrow\left(\mathbb{R}^{+}\right)^{3 g^{-3+b}} \times \mathbb{R}^{3 g^{-3}+b}
$$

be defined by

$$
\Phi_{\mathbf{\alpha}}(\mathscr{F})=\left(\left(m_{j}\right),\left(p_{j}\right)\right) .
$$

The $\left(X_{\alpha}, \Phi_{\alpha}\right)$ are the charts of the piecewise GL $(\cdot, \mathbb{Z} / 4)$-structure.

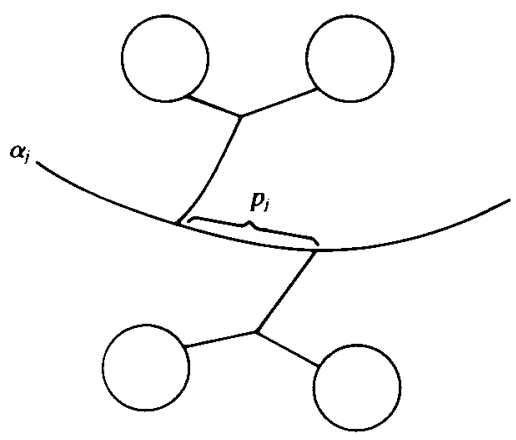

FIGURE 2. Definition of $p_{i}$ given one possible choice of arc and singular prongs.

(1.2) Inductively, $r$ th hitting points of singular prongs of $\mathscr{F}$ on the $\alpha_{j}$ are piecewise linear functions of the $\left(m_{i}, p_{j}\right)$ with coordinates in $\mathbb{Z} / 2$. Since for any $\beta, i(\beta, \mathscr{F})$ can be attained by arranging $\beta$ along segments of singular prong and segments of $\alpha$, the functions

$$
\mathscr{F} \mapsto i(\beta, \mathscr{F})
$$

are piecewise linear, with coefficients in $\mathbb{Z} / 2$.

(1.3) So is $\mathscr{F} \mapsto i(\gamma, \mathscr{F})$, if $\gamma$ is an arc between two boundary components.

(1.4) So is $\mathscr{F} \mapsto i(\gamma, \mathscr{F})$, if $\gamma$ is an arc between two singularities.

(1.5) Let $\gamma_{j}$ be a loop cutting only $\alpha_{j}$ and lying only in the two (or one) adjacent pairs of pants (figure 3). $p_{j}$ is a piecewise linear function of the $m_{k}$, and $i\left(\alpha_{i}, \gamma\right)$, with coefficients in $\mathbb{Z} / 2$. (This is the reason for $\mathbb{Z} / 4$ in the statement of the theorem.)

Theorem 1.1 follows from (1.2), (1.5), since

$$
i\left(\alpha_{j}, \phi \mathscr{F}\right)=i\left(\phi^{-1} \alpha_{j}, \mathscr{F}\right), \quad i\left(\gamma_{j}, \phi \mathscr{F}\right)=i\left(\phi^{-1} \gamma_{j}, \mathscr{F}\right), \quad \text { for } \phi \in \operatorname{Diff}_{0}\left(M_{g, b}\right)
$$




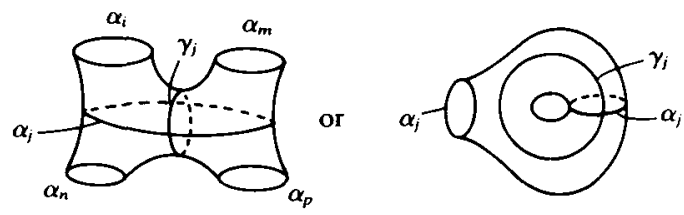

FIGURE 3

\section{Sections of $\mathcal{M} \mathscr{F}$}

Supposed fixed, once and for all, a bounded piecewise smooth (not necessarily globally continuous) section $\mathscr{S} \mathcal{M F}$ of the $\mathbb{R}^{+}$-action on $\mathcal{M F}$.

(1.6) Definition. For $\mathscr{F} \in \mathcal{M} \mathscr{F}$, define $|\mathscr{F}| \in \mathbb{R}^{+}$to be such that $(1 /|\mathscr{F}|) \cdot \mathscr{F} \in \mathscr{P} \mathcal{M} \mathscr{F}$. If $\alpha \in \mathscr{M F}$ is a multiple loop with all components non-intersecting disjoint (from [2, exp. 4], multiple loops can be considered as elements of $\mathscr{M F}$ ), we shall refer to $|\boldsymbol{\alpha}|$ as the length of $\alpha$. $|\mathscr{F}|$ only depends on the choice of $\mathscr{S} \mathcal{M} \mathscr{F}$ up to a bounded proportion.

Note that if $\mathbf{e}$ is any fixed finite set of loops cutting the surface into cells, $|\mathscr{F}|$ is boundedly proportional to $\sum_{j=1}^{N} i\left(e_{j}, \mathscr{F}\right)$ for all $\mathscr{F} \in \mathcal{M F}$, where $\mathbf{e}=\left(e_{1}, \ldots, e_{N}\right)$.

(1.7) Let $\alpha=\left(\alpha_{1}, \ldots, \alpha_{3 g-3+b}\right)$ with all the $\alpha_{i}$ isotopically distinct disjoint. Let

$$
X_{\boldsymbol{\alpha}, \delta}=\left\{\mathscr{F} \in \mathscr{M} \mathscr{F}: i\left(\mathscr{F}, \alpha_{j}\right) \geq \delta|\mathscr{F}|\right\} .
$$

If $\delta$ is small enough, finitely many $X_{\alpha, \delta}$ cover $\mathcal{M} \mathscr{F}$, since the same is true for the $\boldsymbol{X}_{\boldsymbol{\alpha}}$. Note that, if $\boldsymbol{\beta}$ is a loop in $\boldsymbol{X}_{\boldsymbol{\alpha}, \delta}$ and $i(\boldsymbol{\gamma}, \boldsymbol{\beta})=0$, then the integral part of the twist of $\boldsymbol{\gamma}$ round $\alpha_{j}$ is bounded by that of $\boldsymbol{\beta}$, so that $|\gamma|$ can also be estimated by $\sum_{j} i\left(\gamma, \alpha_{j}\right)$. We shall often use this fact. The bound depends only on $\delta$, not on $\beta$.

(1.8) Definition. For $\phi \in \operatorname{Diff}_{0}\left(M_{g, b}\right), \mathscr{F} \in \mathscr{S} \mathscr{M F}\left(M_{g, b}\right)$, define

$$
\boldsymbol{P} \phi(\mathscr{F})=|\phi \mathscr{F}|, \quad \bar{\phi} \cdot \mathscr{F}=\phi \mathscr{F} /|\phi \mathscr{F}| .
$$

Then $(\phi, \mathscr{F}) \mapsto \bar{\phi} \cdot \mathscr{F}$ defines an action of $\operatorname{Diff}_{0}\left(M_{g, b}\right)$ on $\mathscr{S} \mathcal{M F}$, and $(\phi, \mathscr{F}) \mapsto P \phi(\mathscr{F})$ is a cocycle, that is

$$
P(\phi \circ \psi)(\mathscr{F})=P \phi(\bar{\psi} \mathscr{F}) \cdot P \psi(\mathscr{F})
$$

for all $\mathscr{F} \in \mathscr{S} M \mathscr{F}, \phi, \psi \in \operatorname{Diff}_{0}\left(M_{g, b}\right)$.

Note that, if $\mathbf{e}$ is any finite set of loops cutting the surface into cells, both $\sup _{\mathscr{F}_{\in} \in \mathscr{M} \mathscr{M} \mathscr{F}}|\phi \mathscr{F}|, \sup _{\mathscr{F} \in \mathscr{C}, \mathcal{M} F}\left|\phi^{-1} \mathscr{F}\right|$ are boundedly proportional to

$$
\sum_{j, k=1}^{N} i\left(\phi e_{j}, e_{k}\right)=\sum_{j, k=1}^{N} i\left(\phi^{-1} e_{j}, e_{k}\right),
$$

for all $\phi \in \operatorname{Diff}_{0}\left(\boldsymbol{M}_{g, b}\right)$, where $e=\left(e_{1}, \ldots, e_{N}\right)$. For, if $\boldsymbol{\alpha}$ is a multiple loop,

$$
i\left(\phi \boldsymbol{\alpha}, e_{k}\right)=i\left(\boldsymbol{\alpha}, \phi^{-1} e_{k}\right) .
$$

Then $\alpha$ can be arranged to lie only along arcs of the $e_{j}$ 's with $\leq D|\boldsymbol{\alpha}|$ segments along each $e_{j}$. So

$$
i\left(\phi^{-1} e_{k}, \boldsymbol{\alpha}\right) \leq C_{1}|\boldsymbol{\alpha}| \sum_{j} i\left(e_{j}, \phi^{-1} e_{k}\right)
$$


and

$$
|\phi \boldsymbol{\alpha}| \leq C_{2} \sum_{k} i\left(\phi \boldsymbol{\alpha}, e_{k}\right) \leq C_{1} C_{2}|\boldsymbol{\alpha}| \sum i\left(e_{j}, \phi^{-1} e_{k}\right)
$$

For general $\mathscr{F}$, the result follows from piecewise continuity of $i(1.1)$, and density of multiple loops.

We have a natural Lebesgue measure on $\mathscr{S} \mathscr{M} \mathscr{F}$, up to bounded Radon-Nikodym derivative, which we denote also by $\lambda$ (as well as the measure on $\mathcal{M F}$ ).

(1.9) LemMA.

$$
\frac{1}{C} \leq\left|\frac{d \bar{\phi}_{*} \lambda}{d \lambda}(\mathscr{F})\right| \cdot|P \phi(\mathscr{F})|^{6 \mathrm{~g}-6+2 b} \leq C
$$

for all $\phi, \mathscr{F}$, for some constant $C$, where $\bar{\phi}_{*} \lambda(A)=\lambda(\bar{\phi} A)(A \subseteq \mathscr{S} \mathcal{M F})$.

Proof. Let $(a, b) \cdot A$ denote $\{t \cdot A: t \in(a, b)\}$. Use the facts that:

$$
\lambda((a, b) \cdot A)=\int_{a}^{b} t^{6 z-7+2 b} d t \cdot \lambda(A),
$$

where the left-hiand $\lambda$ is measure on $\mathscr{M F}$, right-hand $\lambda$ is measure on $\mathscr{S} \mathscr{M F}$; for $\lambda$ on $\mathcal{M F}$, by (1.1), the $d \phi_{*} \lambda / d \lambda$ are uniformly bounded; and

$$
P \phi(t \mathscr{F})=t \cdot P \phi(\mathscr{F}) \text {. }
$$

In summary. $\mathscr{S} \mathcal{M F}$ can be identified with the $\mathbb{R}^{+}$-orbit space of $\mathscr{M F}$. One of the two main objects of the present paper is the study of the ergodic theory of $\left(\mathscr{S} \mathcal{M} \mathscr{F}, \operatorname{Diff}_{0}\left(M_{g, b}\right), \lambda\right)$.

\section{The intersection number function}

For any two multiple loops $\alpha, \beta$ on a surface $M_{g, b}$ (for which the components of $\boldsymbol{\alpha}$ do not intersect each other, and similarly for $\boldsymbol{\beta}) i(\boldsymbol{\alpha}, \boldsymbol{\beta})$ is defined as the minimal number of intersections of loops $\boldsymbol{\alpha}^{\prime}, \boldsymbol{\beta}^{\prime}$ isotopic to $\boldsymbol{\alpha}, \boldsymbol{\beta}$ respectively.

For $\alpha$ a multiple loop, and $\mathscr{F} \in \mathcal{M} \mathscr{F}, i(\alpha, \mathscr{F})$ is defined as the minimal $\mathscr{F}$-measure of a loop in the isotopy class of $\alpha$. If $\mathscr{F}$ is itself a multiple loop, this definition coincides with the previous one.

$\left\{\lambda \boldsymbol{\alpha}: \lambda \in \mathbb{R}^{+}, \boldsymbol{\alpha}\right.$ a multiple loop $\}$ is dense in $\mathscr{M F}$ (since, for example, in a chart $\Phi_{\beta}\left(X_{\beta}\right)$, any point for which the $m_{i}$-coordinates are even integers, and the $p_{i}$ coordinates are integers, is a multiple loop). It is proved in [2] that $\mathscr{F} \mapsto i(\alpha, \mathscr{F})$ is continuous on $\mathscr{M F}$. We prove:

(1.10) LEMMA. $\mathscr{F} \rightarrow i(\boldsymbol{\alpha}, \mathscr{F})$ is Lipschitz with constant $C|\boldsymbol{\alpha}|$, some universal $C$, with respect to any metric in the natural class of Lipschitz-equivalent metrics on $\mathcal{M F}$ (given, for example, by Euclidean-norm-metrics on the charts $\Phi_{\alpha}\left(X_{\alpha}\right)$ of (1.1)).

Proof. It suffices to prove the function is Lipschitz restricted to a set of $\mathscr{F}$ in an $X_{\beta, \delta}(1.7)$ all with the same picture (as in (1.1)) with respect to $\beta$. Fix a metric $d$ on $\mathcal{M F}$. Let $\boldsymbol{\alpha}$ be such that $\mu_{\mathscr{F}}(\boldsymbol{\alpha})=i(\boldsymbol{\alpha}, \mathscr{F})$.

Let $\alpha$ cross $\beta_{i}$ at points $x_{i, 1}, \ldots, x_{i, j}$, where $x_{i, k}$ varies between 0 and $m_{i}$, and these coordinates on $\beta_{i}$ are with respect to an origin which we have fixed as the first 
hitting point of a singular prong of $\mathscr{F}$. If, for nearby $\mathscr{F}^{\prime}$, we arrange $\alpha$ to cross $\beta$ at points

then we clearly have

$$
\frac{x_{i k}}{m_{i}(\mathscr{F})} m_{i}\left(\mathscr{F}^{\prime}\right)
$$

$$
i(\boldsymbol{\alpha}, \mathscr{F}) \leq \mu_{\mathscr{F} \prime}(\boldsymbol{\alpha}) \leq C^{\prime}|\boldsymbol{\alpha}| d\left(\mathscr{F}, \mathscr{F}^{\prime}\right)+i(\boldsymbol{\alpha}, \mathscr{F}),
$$

some $C^{\prime}$. By symmetry,

$$
\left|i(\boldsymbol{\alpha}, \mathscr{F})-i\left(\boldsymbol{\alpha}, \mathscr{F}^{\prime}\right)\right| \leq C|\boldsymbol{\alpha}| d\left(\mathscr{F}, \mathscr{F}^{\prime}\right) .
$$

As an immediate corollary, we obtain the following unpublished result of Thurston:

(1.11) COROLlaRY. $i$ extends to a continuous Lipschitz function $\mathcal{M F} \times \mathcal{M} \mathscr{F} \rightarrow \mathbb{R}^{+}$ satisfying

$$
i\left(\mathscr{F}_{1}, \mathscr{F}_{2}\right)=i\left(\mathscr{F}_{2}, \mathscr{F}_{1}\right), \quad i\left(\lambda \mathscr{F}_{1}, \mathscr{F}_{2}\right)=\lambda i\left(\mathscr{F}_{1}, \mathscr{F}_{2}\right)
$$

Proof. $i(\lambda \alpha, \mathscr{F})$ is already defined and, by (1.10), is Lipschitz with constant $\leq C \lambda|\boldsymbol{\alpha}|$. Let $\lambda_{n} \alpha_{n} \rightarrow \mathscr{G}$. By Ascoli's theorem, since $\left|\lambda_{n} \boldsymbol{\alpha}_{n}\right|$ is bounded, and hence $i\left(\lambda_{n} \boldsymbol{\alpha}_{n}, \mathbf{e}\right)$ is bounded for any fixed multiple loop, some subsequence of $i\left(\lambda_{n} \boldsymbol{\alpha}_{n}, \cdot\right)$ converges to a Lipschitz function $g$, with constant $\leq C_{1}|\mathscr{G}|$, some $C_{1}$. Also

$$
g(\mu \boldsymbol{\beta})=i(\mu \boldsymbol{\beta}, \mathscr{G})
$$

for all multiple loops $\boldsymbol{\beta}$, all $\mu \in \mathbb{R}^{+}$, since

$$
i\left(\lambda_{n} \boldsymbol{\alpha}_{n}, \mu \boldsymbol{\beta}\right)=i\left(\mu \boldsymbol{\beta}, \lambda_{n} \boldsymbol{\alpha}_{n}\right) \rightarrow i(\mu \boldsymbol{\beta}, \mathscr{G}) .
$$

Hence, since scalar multiples of multiple loops are dense, $\left\{i\left(\lambda_{n} \boldsymbol{\alpha}_{n}, \cdot\right): n \in \mathbb{N}\right\}$ has only one limit point, the sequence actually converges, and we can denote the limit by $i(\mathscr{G} \cdot)$.

\section{Minimal foliations and the cone of invariant measures}

A measured foliation on $M_{g, b}$ is defined to be minimal if all leaves are either dense in $M_{g, b}$, or join two singularities - and there must be no cycle of leaves of the latter type. It is well known that an $\mathscr{F} \in \mathcal{M F}$ is minimal if and only if it is irreducible, that is, there is no cycle of leaves (see [1], for instance). A non-minimal foliation can thus be regarded as a foliation of a subsurface - the set of these is a countable union of subvarieties of lower dimension than the dimension of $\mathcal{M F}\left(\boldsymbol{M}_{8, b}\right)$, and hence has zero Lebesgue measure.

It is also well known that the set of invariant measures for $\mathscr{F} \in \mathcal{M} \mathscr{F}\left(\boldsymbol{M}_{g, b}\right)$ is of finite dimension $<6 g-6+2 b$. In fact, by (1.2), the 'configuration' of an $\mathscr{F} \in X_{\beta}$ (1.1) and the coordinates $m_{i}(\mathscr{F}), p_{i}(\mathscr{F})$ with respect to $\beta$, determine the measure between any two hitting points of singular prongs of $\mathscr{F}$ on $\boldsymbol{\beta}$. By minimality, these give a generating partition on $\boldsymbol{\beta}$. So any transverse invariant measure on $\mathscr{F}$ is determined by coordinates $m_{i}^{\prime}, p_{i}^{\prime}$, which can be identified with another measured foliation $\mathscr{F}^{\prime}$.

This identifies the cone of invariant measures for $\mathscr{F}$ with a 'subcone' of $\mathscr{M F}$. This is, in fact, all we need for proving our theorems, but it may be interesting to 
prove the following, which was first explained to me by Laudenbach in terms of geodesic laminations.

(1.12) THEOREM. Let $\left(\mathscr{F}, \mu_{\mathscr{F}}\right)$ be minimal, and $\left(\mathscr{F}^{\prime}, \mu_{\mathscr{F F}}\right)$ another measured foliation. The following are equivalent.

(1) $\mathscr{F}, \mathscr{F} '$ are Whitehead equivalent, that is, isotopic after some singularities joined by prongs have possibly been amalgamated, and the reverse has possibly happened for other singularities.

(2) $i\left(\mathscr{F}, \mathscr{F}^{\prime}\right)=0$.

Proof. $1 \Rightarrow 2$. Write $\left(\mathscr{F}, \mu_{\mathscr{F}}\right)=\left(\mathscr{F}, \mu_{1}\right)$. Since $\mathscr{F}, \mathscr{F}$ are Whitehead equivalent, as a measured foliation $\left(\mathscr{F}^{\prime}, \mu_{\mathscr{F}}\right)=\left(\mathscr{F}, \mu_{2}\right)$, some $\mu_{2}$ not necessarily equivalent to Lebesgue measure. Let $\left\{\alpha_{n}\right\}$ be a sequence of multiple loops, and $\left\{\lambda_{n}\right\} \subseteq \mathbb{R}^{+}$such that $\lambda_{n} \alpha_{n} \rightarrow \mathscr{F}$. Then, by (1.11), $i\left(\lambda_{n} \alpha_{n}, \mathscr{F}\right) \rightarrow 0$. We aim to show $i\left(\lambda_{n} \alpha_{n}, \mathscr{F}^{\prime}\right) \rightarrow 0$, so that, by $(1.11), i\left(\mathscr{F}, \mathscr{F}^{\prime}\right)=0$.

Choose e so that $\mathscr{F}, \mathscr{F}^{\prime} \in X_{\mathrm{e}}$. We may arrange $\alpha_{n}$ to lie piecewise along and leaves of $\mathscr{F}$, such that

$$
\mu_{1}\left(\alpha_{n}\right)=i\left(\alpha_{n}, \mathscr{F}\right), \quad \mu_{2}\left(\alpha_{n}\right)=i\left(\alpha_{n}, \mathscr{F}^{\prime}\right) .
$$

Split up any segment of $\alpha_{n}$ winding round an $e_{i}$ several times into subsegments winding round at most once, so that $\alpha_{n}$ consists of $\mathscr{F}$-leaf segments and $\leq C_{1}\left|\alpha_{n}\right|$ segments of e all with $\mu_{2}$-measure $\leq C_{2}$. Also,

$$
C_{3} /\left|\alpha_{n}\right| \leq \lambda_{n} \leq C_{4} /\left|\alpha_{n}\right|
$$

since $\lambda_{n} \alpha_{n} \rightarrow \mathscr{F}$.

Given $\delta$, choose $N$ such that

$$
\lambda_{n} i\left(\alpha_{n}, \mathscr{F}\right)<\delta^{2}, \quad n \geq N .
$$

Then

$$
\mu_{1}\left(\alpha_{n}\right)=i\left(\alpha_{n}, \mathscr{F}\right) \leq \delta^{2}\left|\alpha_{n}\right| / C_{3} .
$$

If $R$ of the segments of $\alpha_{n}$ along e have $\mu_{1}$-measure $\geq \delta$, then $R \leq \delta C_{2}\left|\alpha_{n}\right|$. Both $\mu_{1}, \mu_{2}$ are non-atomic (since $\mathscr{F}$ is minimal) so, given $\varepsilon$, we can find $\delta$ such that any segment of e with $\mu_{1}$-measure $<\delta$ has $\mu_{2}$-measure $<\varepsilon$. Thus

$$
\mu_{2}\left(\alpha_{n}\right) \leq R C_{2}+C_{1}\left|\alpha_{n}\right| \varepsilon
$$

Hence

$$
i\left(\lambda_{n} \alpha_{n}, \mathscr{F}^{\prime}\right)=\lambda_{n} \mu_{2}\left(\alpha_{n}\right) \leq C_{1} C_{2} C_{4} \delta+C_{1} C_{4} \varepsilon,
$$

and $i\left(\lambda_{n} \alpha_{n}, \mathscr{F}^{\prime}\right) \rightarrow 0$, as required.

$2 \Rightarrow 1$. Suppose $i\left(\mathscr{F}, \mathscr{F}^{\prime}\right)=0$. Choose $\left\{\alpha_{n}\right\}$ a sequence of multiple loops, and $\left\{\lambda_{n}\right\} \subseteq \mathbb{R}^{+}$such that

$$
d\left(\lambda_{n} \alpha_{n}, \mathscr{F}^{\prime}\right) \leq C_{1} /\left|\alpha_{n}\right|^{\delta+1}, \quad \lambda_{n} \geq C_{2} /\left|\alpha_{n}\right|, \quad \delta=1 /(6 g-7+2 b),
$$

assuming without loss of generality that $\mathscr{F}^{\prime} \in \mathscr{S} \mathscr{M F}$, and $d$ is some fixed Lipschitz metric on $\mathcal{M F}$. This is possible because a point in $\mathbb{R}^{m}$ can be approximated to within 
$N^{-1-1 / m}$ by a rational of the form $\left(p_{1} / N, \ldots, p_{m} / N\right)$ for infinitely many $N$. Then

$$
i\left(\alpha_{n}, \mathscr{F}\right) \leq C_{3} /\left|\alpha_{n}\right|^{\delta},
$$

by (1.11) $\lambda_{n} \alpha_{n} \rightarrow \mathscr{F}$, and we aim to show that, in the limit, $\lambda_{n} \alpha_{n}$ defines an $\mathscr{F}$-invariant tranverse measure. Let e be a fixed multiple loop with $\mathscr{F}, \mathscr{F}^{\prime} \in X_{\mathbf{e}}(1.1)$. Arrange $\alpha_{n}$ to lie only along singular leaves of $\mathscr{F}$ and arc segments of e. Since $i\left(\alpha_{n}, \mathscr{F}\right) \rightarrow 0$, for large $n$, all segments of $\alpha_{n}$ must follow a leaf of $\mathscr{F}$ through $N$ pairs of pants for $N$ large. Further, except for a union of segments of $\alpha_{n}$ with $\leq_{\varepsilon}\left|\alpha_{n}\right|$ intersection points with e, all points of $\alpha_{n} \cap \mathbf{e}$ lie in segments of $\alpha_{n}$ which follow some leaf of $\mathscr{F}$ both forwards and backwards through $N$ pairs of pants, for $n$ sufficiently large.

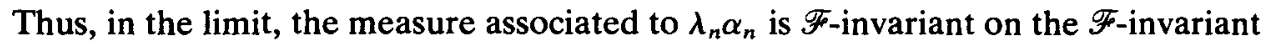
partition of e given by the different isotopy paths that leaves of $\mathscr{F}$ can take through $N$ pairs of pants, for all $N$. This partition is generating, since $\mathscr{F}$ is minimal, and the endpoints of the intervals of the partition are precisely the hitting points of singular prongs. So the only isotopy paths through $N$ pairs of pants which occur for $\mathscr{F}$, occur for $\mathscr{F}^{\prime}$, and $\mu_{\mathscr{F}^{\prime}}$ is $\mathscr{F}$-invariant.

We now show that $\mathscr{F}, \mathscr{F}$ ' are isotopic, assuming as we may do that they are both transverse to $e$ and have the same type of singularities in each pair of pants (because they have the same isotopy paths). Let $\Phi$ be defined as mapping hitting points of singular leaves of $\mathscr{F}$ on e to the corresponding ones for $\mathscr{F}^{\prime} . \Phi$ is well-defined and order-preserving, since $\mu_{\mathscr{F}}$ is $\mathscr{F}$-invariant. Hitting points for $\mathscr{F}$ are dense by minimality, and also for 'Fु', since otherwise $\mu_{\mathscr{F}}$ would have an atomic part. So $\Phi$ extends uniquely to $e$ and is a homeomorphism, since its inverse can be defined by the same argument. $\Phi$ extends to a homeomorphism of the whole surface mapping leaves of $\mathscr{F}$ to leaves of $\mathscr{F}^{\prime}$. (The only obstruction would be if $\mathscr{F}, \mathscr{F}^{\prime}$ had different integral twists round some $e_{i}$-but this cannot happen, since isotopy paths

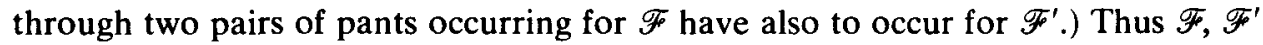
are isotopic.

We end this section with the following well-known fact about minimal foliations, which we shall need in $\S 3$.

(1.13) Lemma [1, §2]. Let $\mathscr{F}$ be a minimal foliation. Assume, without loss of generality, that $\mathscr{F}$ defines a flow (otherwise take the oriented double cover). Let I be a fixed transversal and $T_{\mathscr{F}}$ the first return map to $I$. Then $T_{\mathscr{F}}$ is a (finite) interval exchange, and for any open $U \subseteq I$ there exists $N$ such that $U \cup \cdots \cup T_{\$}^{N} U=I$.

In fact, this is an easy consequence of Poincare recurrence and the fact that the number of singularities is finite.

\section{Multiple loops and the associated elements of Diff $f_{0}$} We continue with the notations established in $\S 1$.

(2.1) Definitions. As usual, a multiple loop $\alpha$ on a surface is a finite set of nonintersecting non-self-intersecting loops. A multiple loop $\alpha$ is maximal if no loop non-isotopic to all components of $\alpha$ has zero intersection with $\alpha$. On $M_{g, b}$ this 
means $\alpha$ has components in $3 g-3+b$ distinct isotopy classes. A multiple loop is prime if it has at most one component in any isotopy class.

The aim of this section is to prove, first:

(2.2) Proposition. Given $M_{\mathrm{g}, b}$, there exist constant $A, B, C$ such that the number of prime maximal loops of length $(1.6) \geq A^{n},<A^{n+1}$ with all components of length $\geq C A^{n}$, is $\geq C A^{(6 g-6+2 b) n}$ and $\leq B A^{(6 g-6+2 b) n}$.

We want this in order to prove a result about $\operatorname{Diff}_{0}\left(M_{g, b}\right)$ (proposition (2.10)). Note that the upper bound on cardinality in proposition (2.2) is trivial since it holds for multiple loops - because under $\Phi_{\alpha}(1.1)$ multiple loops map to a set containing $(2 \mathbb{N})^{3 g-3+b} \times \mathbb{Z}^{3 g-3+b}$ and contained in $(\mathbb{N})^{3 g-3+b} \times \mathbb{Z}^{3 g-3+b}$. We shall also need a similar result to (2.2) for certain kinds of orientable loops (for the interval exchange theorem) for which the proof is only a little different.

Suppose we are given a prime multiple loop e on $M_{\mathrm{g}, 0}$ cutting the surface into holed spheres, together with an orientation on each component of e. Let $\mathscr{L}$ denote a set of oriented foliations transverse to e, with one singularity in each holed sphere, the orientation defined by leaves intersecting with e being constant, and the configuration of singular prongs up to first intersections with e being constant on $\mathscr{L}$. Suppose $\mathscr{L}$ consists of all the foliations with these fixed configurations. For instance, the configuration in one holed sphere might be fixed as shown in figure 4. $\mathscr{L}$ is then a subvariety of $\mathscr{M F}\left(M_{g, 0}\right)$ with constant dimension - because in each

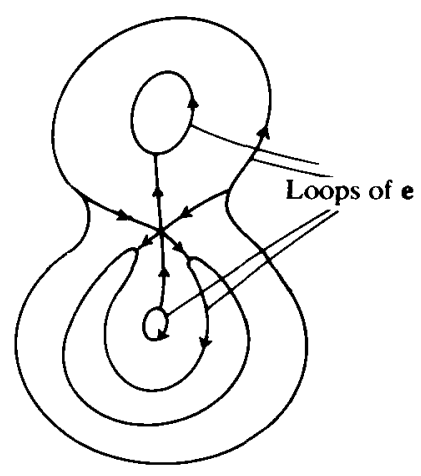

FIGURE 4

holed sphere, the foliation is defined by the measure of pieces of boundary, and to obtain a foliation on all $M_{\mathrm{g}, 0}$, we only need equalities between the two different measures of each $e_{i}, \mathbf{e}=\left(e_{1}, \ldots, e_{p}\right)$. Maximal loops in $\mathscr{L}$ are those to which no new isotopy classes can be added to obtain another loop in $\mathscr{L}$.

(2.3) Proposition. Proposition (2.2) holds for prime maximal loops in $\mathscr{L}$ instead of $\mathscr{M} \mathscr{F}\left(M_{\mathrm{g}, b}\right)$, with $6 \mathrm{~g}-6+2 b$ replaced by $s=\operatorname{dim} \mathscr{L}$.

The idea of the proof of these two propositions is to modify arbitrary maximal loops to obtain loops with the required properties. To start, we need: 
(2.4) LeMMA. Given $M_{g, b}$, there exists a constant $D$ such that any laop $\alpha$ can be completed to a maximal loop $\boldsymbol{\alpha}^{\prime}$ with $\left|\boldsymbol{\alpha}^{\prime}\right| \leq D|\boldsymbol{\alpha}|$.

Proof. Fix a prime maximal loop e with $\alpha \in X_{\mathrm{e}, \delta}$ (1.7). $M_{g, b}-\alpha$ has components $S_{1}, \ldots, S_{N}$ which are not merely cylinders homotopic to components of $\alpha, N<$ $2 g-2+b$. e cuts each $S_{i}$ into $\leq D_{2}|\boldsymbol{\alpha}|$ pieces. For instance, $M_{2,0}-\left\{\alpha_{1}, \alpha_{2}\right\}$ might have one component as in figure 5 .

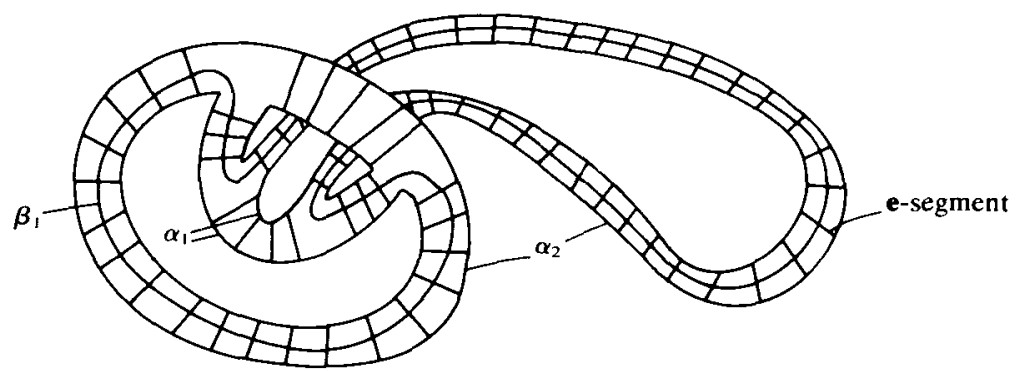

FigURE 5

If we erase all those segments of $e$ just bordering on rectangles, we arrive at a finite set (depending only on $\delta$ ) of partial triangulations of $S_{i}$. For each $S_{i}$, choose a maximal loop set $\boldsymbol{\beta}_{i}$ in $S_{i}$ with no component isotopic to a boundary component (see figure 5, for example) and let $\boldsymbol{\alpha}^{\prime}$ be obtained from $\boldsymbol{\alpha}$ by adjoining $\boldsymbol{\beta}_{1}, \ldots, \boldsymbol{\beta}_{N}$. There exists $D_{3}$ such that $\boldsymbol{\beta}_{i}$ passes through each region of the triangulation $\leq D_{3}$ times, hence crosses e $\leq D_{2} D_{3}|\alpha|$ times.

$$
\left|\boldsymbol{\alpha}^{\prime}\right| \leq D_{4} i\left(\boldsymbol{\alpha}^{\prime}, \mathbf{e}\right)
$$

and so

$$
\left|\boldsymbol{\alpha}^{\prime}\right| \leq D|\boldsymbol{\alpha}| .
$$

Remark. Modifications of (2.4) needed towards (2.3) are trivial.

The following can be proved using the same method as (2.4).

(2.4') Lemma. Let $\alpha=\left(n_{1} \alpha_{1}, \ldots, n_{s} \alpha_{s}\right)$ be any multiple loop. There exists a simple $\beta$ such that $\beta$ crosses each $\alpha_{i}$ at least once, but $\leq D$ times, and $|\beta| \leq D|\boldsymbol{\alpha}|$, for $D$ independent of $\boldsymbol{\alpha}$.

Proof. As in (2.4), obtain a partial triangulation of $M_{g, b}-\boldsymbol{\alpha}$, hence a partial triangulation of $M_{g, b}$ by adding the $\alpha$-decomposition. There is only a finite set of possible triangulations (up to homeomorphism) so a loop $\beta$ can be drawn crossing the edges of the triangulation $\leq D_{1}$ times (and each $\alpha_{i}$ at least once), which gives $|\beta| \leq D|\boldsymbol{\alpha}|$ for suitable $D$. (A slight modification of (1.7) is used - clearly the twist of $\beta$ around e cannot be much greater than that for $\boldsymbol{\alpha}$.) 
(2.5) In the proofs of (2.2), (2.3), we shall use the following principle without further explanation. Let $\mathscr{S}_{n}$ be a set of loops $\alpha$ with

$$
A^{n} \leq|\boldsymbol{\alpha}|<A^{n+1}
$$

and

$$
\#\left(\mathscr{S}_{n}\right) \geq C_{1} A^{n},
$$

some $C_{1} \leq 1, A>1$. Let $F$ be a map of $\bigcup_{n} \mathscr{P}_{n}$ into multiple loops with

$$
C_{2}|\mathbf{\alpha}| \leq|F(\boldsymbol{\alpha})| \leq C_{3}|\boldsymbol{\alpha}| \quad\left(C_{2}<1, C_{3}>1\right)
$$

and $F^{-1} F(\boldsymbol{\alpha})$ having $\leq C_{4}$ elements for all $\alpha$. Then for $A_{1}=A^{2}\left(C_{3} / C_{2}\right)^{2}$, the number of elements $\alpha$ of $\operatorname{Im}(F)$, with $A_{1}^{n} \leq|\boldsymbol{\alpha}|<A_{1}^{n+1}$ is $\geq\left(1 / C_{4}\right) A_{1}^{n}$, because we can find, for any $n$, an $r$ such that

$$
\left[r \cdot \log A-\log \left(C_{3} / C_{2}\right),(r+1) \log A+\log \left(C_{3} / C_{2}\right)\right] \subseteq\left[n \cdot \log A_{1},(n+1) \log A_{1}\right]
$$

Proof of (2.2). By (2.5), it suffices to construct a map $F$ from the set of multiple loops into the set of prime maximal loops

$$
\boldsymbol{\alpha}=\left(\alpha_{1}, \ldots, \boldsymbol{\alpha}_{3 \mathrm{~g}-3+b}\right)
$$

with $\left|\boldsymbol{\alpha}_{i}\right| \geq C|\boldsymbol{\alpha}|$ for all $i$, such that

$$
L|\boldsymbol{\beta}| \leq|F(\boldsymbol{\beta})| \leq K|\boldsymbol{\beta}|,
$$

and $F^{-1} F(\boldsymbol{\beta})$ having $\leq H$ elements for all $\boldsymbol{\beta}$, suitable $H, K, L$. In fact, we shall construct $F$ as a composition $F_{4} \circ F_{3} \circ F_{2} \circ F_{1}$, where

$$
L_{i}|\boldsymbol{\beta}| \leq\left|F_{i}(\boldsymbol{\beta})\right| \leq K_{i}|\boldsymbol{\beta}|
$$

and $F_{i}^{-1} F_{i}(\boldsymbol{\beta})$ has $\leq H_{i}$ elements, for all $\boldsymbol{\beta}$.

$F_{1}$ maps multiple loops to maximal loops. $F_{1}$ exists with the required properties by (2.4).

$F_{2}$ maps $\alpha=\left(n_{i} \alpha_{i}\right)$ to

$$
\left(\left(n_{i}+[|\boldsymbol{\alpha}|] /\left[\left|\boldsymbol{\alpha}_{i}\right|\right]\right) \cdot \boldsymbol{\alpha}_{i}\right)=\left(n_{i}^{\prime} \boldsymbol{\alpha}_{i}\right),
$$

where $[x]$ denotes the integral part of $x$. So

$$
\left|n_{i}^{\prime} \alpha_{i}\right| \geq M\left|F_{2}(\boldsymbol{\alpha})\right|
$$

some constant $M$.

$F_{3}$ maps $\operatorname{Im}\left(F_{2}\right)$ into the simple loops as follows. For $\alpha \in \operatorname{Im}\left(F_{2}\right)$, let $\beta_{\alpha}$ be as $\beta$ in $\left(2.4^{\prime}\right)$. Note that we can, and do, choose $\beta_{\alpha}$ so that $\left|\beta_{\alpha}\right|$ is minimal among the lengths of all loops obtained by twisting $\beta_{\alpha}$ around the $\alpha_{i}$, if $\alpha=\left(n_{i} \alpha_{i}\right)$. (All such loops cross all $\alpha_{i}$ at most $D$ times, $D$ as in $\left(2.4^{\prime}\right)$.) Let $F_{3}(\alpha)$ be obtained by twisting $\beta_{\alpha}\left(n_{i}+D+3\right)$ times round each $\alpha_{i}$. (It does not matter in which direction we twist.) The necessary upper bound on $F_{3}(\alpha)$ is clear. The lower bound holds because no twist round an $\alpha_{i}$ can be unwound more than once at each end, so $F_{3}(\boldsymbol{\alpha})$ has at least the order of magnitude of all the $\left|n_{i} \alpha_{i}\right|$, which are all of the order of $|\boldsymbol{\alpha}|$. Also, any loop not intersecting $F_{3}(\boldsymbol{\alpha})$ must cross at least one $\alpha_{i}$ (because $\boldsymbol{\alpha}$ is maximal), hence must wind round $\alpha_{i}$ at least $n_{i}+1$ times, hence have order of magnitude $\geq|\boldsymbol{\alpha}|$. If we take $F_{4}\left(F_{3}(\alpha)\right)$ to be a maximal loop obtained by $(2.4), F_{4} F_{3}(\boldsymbol{\alpha})$ will have all components of the same magnitudes, as required. It remains to show that $F_{3}$ is boundedly finite-ro-one. 
The idea is that $n_{i}, \alpha_{i}$ can be reconstructed from $F_{3}(\alpha)$. Each $\alpha_{i}$ has the property that there are between $R\left(n_{i}+D+2\right)$ and $R\left(n_{i}+D^{\prime}+4\right)$ adjacent strands of $F_{3}(\alpha)$ (some $R \leq D$ ) such that the loop traced by $F_{3}(\alpha)$ between one strand and another one $R$ strands away is $\alpha_{i}$ (figure 6). No other loop, apart from the $\alpha_{i}$, has this property with respect to $F_{3}(\alpha)$, with $n_{i}$ replaced by any number $\geq 1$. For if there were such a loop, we could reverse the twists round the $\alpha_{i}$ and obtain a loop with this property with respect to $\beta_{\alpha}$ instead of $F_{3}(\alpha)$. But no loop can be traced $D+1$ times by $\beta_{\alpha}$ - because $\beta_{\alpha}$ crosses no $\alpha_{i}$ more than $D$ times.

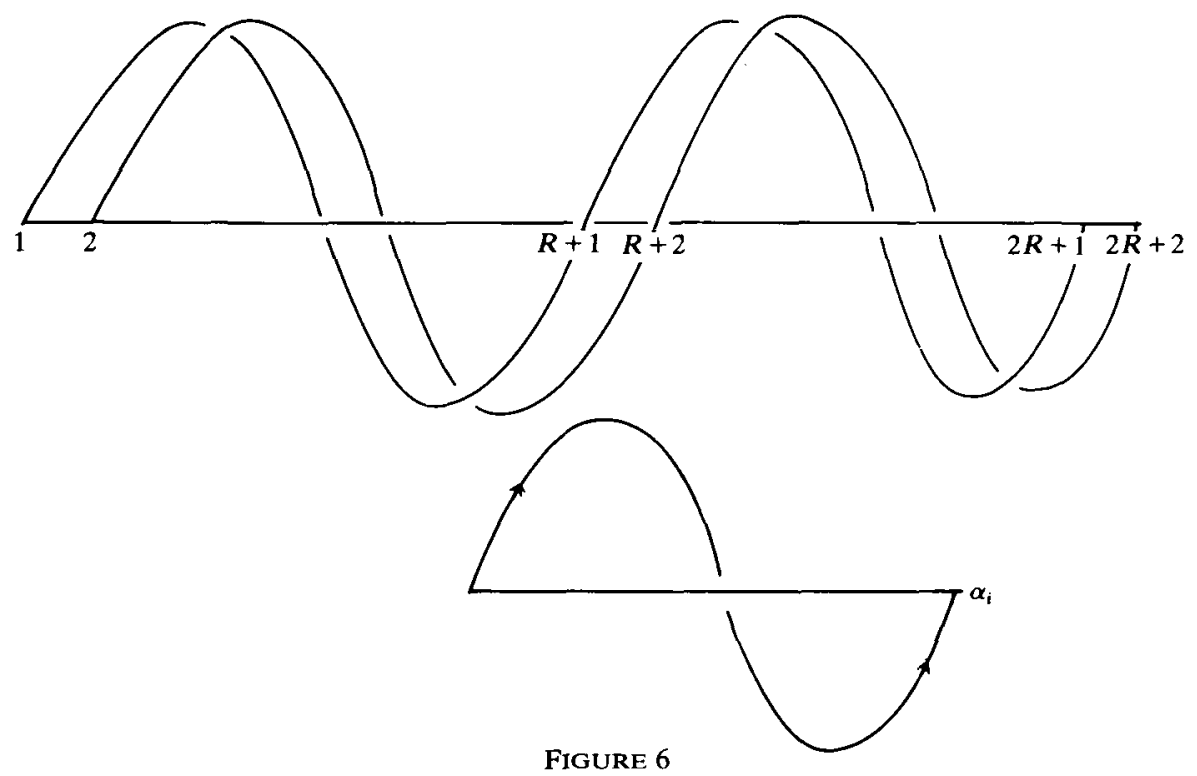

For the proof of (2.3) we need (2.6), (2.7), and (2.8), of which we omit the complete proofs.

(2.6) Note that for $\mathscr{L} \subseteq \mathcal{M F}\left(M_{\mathrm{g}, 0}\right)$ as described, an $\alpha \in \mathscr{L}$ has no component separating $M_{\mathrm{g}, 0}$ - for a component $\alpha_{i}$ of $\alpha$ in $\mathscr{L}$ is oriented, and as shown in figure 7 , restricted to a holed sphere in $\boldsymbol{M}_{\mathrm{g}, 0}-\mathbf{e}$, it is possible to travel from a 'left' side of a segment of $\alpha_{i}$ to a 'right' side without crossing $\alpha$-which means that the 'left' side surface is the same as the 'right' side surface, and $\alpha_{i}$ does not separate.
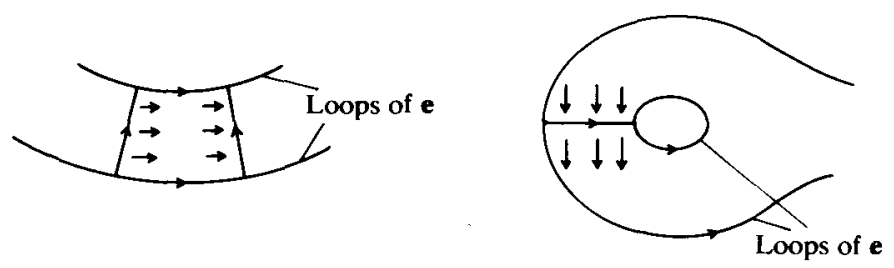

FIGURE 7

(2.7) LeMma. A loop $\alpha$ in $\mathscr{L}$ has a maximal number of distinct isotopy components if and only if all components of $M_{g, 0}-\alpha$ are holed spheres containing one singularity each. 
Idea of proof. A component of $M_{g, 0}-\alpha$ is cut by $\mathrm{e}$ into star shapes, each containing one singularity, and with an even number of (square-ended) points (figure 8). The orientation on e, and on $M_{g, 0}$, assigns a direction to each prong in each star, in

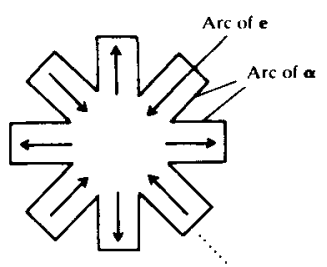

FIGURE 8

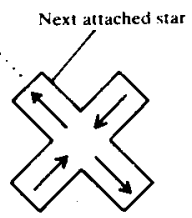

which a loop of $\mathscr{L}$ lying in $M_{g, 0}-\alpha$ must travel. The directions alternate round singularities. When a loop arrives at a singularity, it can only pass into the adjacent prongs. It suffices to show that if there is more than one singularity or if there is homology, then there is a loop of $\mathscr{L}$ in $M_{g, 0}-\alpha$ non-isotopic to a component of $\alpha$. In the case of 2 singularities we proceed as shown in figure 9.

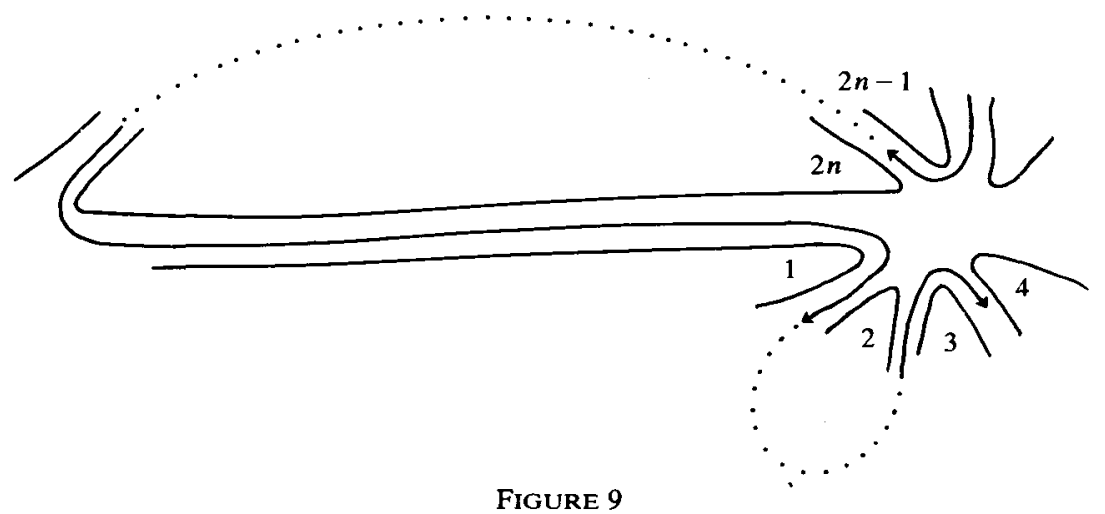

Moving straight to tracing boundary component $2 r$, if in tracing round component $2 s, s<r$, we arrive at the corner of $2 r-1$. We simply claim that, with such a rule, the loop is never forced to intersect itself. The same lemma occurs, with different language, in [5].

(2.8) LEMMA. If a component of $M_{g, 0}-\boldsymbol{\alpha}(\boldsymbol{\alpha} \in \mathscr{L})$ is a holed sphere with one singularity, a non-self-intersecting path can be drawn from one point in the component to any other, without crossing the singularity, and respecting the orientation.

Idea of proof. The configuration of prongs is something like figure 10. In words, if prongs $x$ and $y$ are identified, and $z$ and $w$ are identified, then $z, w$ lie on 'the same' side of $x, y$ (because attaching handles cannot decrease genus). 


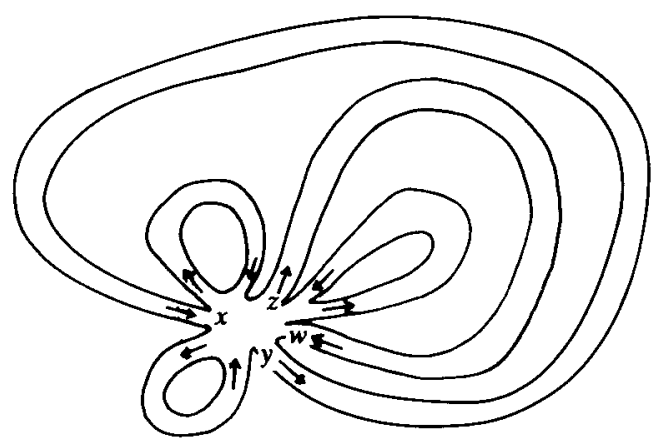

Figure 10

Suppose starting at point $a$, you want to arrive at $b$. Each time you arrive at the singularity, you have a choice of two prongs. Choose the one 'closest' to $b$. Eventually, you arrive at $b$.

Proof of (2.3). The idea is the same as for (2.2). $F_{1}, F_{2}$ are constructed as before. But $F_{4} \circ F_{3}$ is replaced by a composition $F_{4, s} \circ F_{3, s} \circ \cdots \circ F_{4,1} \circ F_{3,1} . \operatorname{Im}\left(F_{4, \ell}\right)$ has the property that $\alpha \in \operatorname{Im}\left(F_{4, t}\right)$ is maximal, and has at least $t$ isotopy components occurring singly and of the same order of magnitude as $\alpha$.

Assume $F_{3, i}, F_{4, i}$ have been constructed, $i \leq t$. Define

$$
F_{3, t+1}(\boldsymbol{\alpha})=F_{4, t+1}(\boldsymbol{\alpha})=\left(\alpha_{1}, \ldots, \alpha_{s}\right),
$$

if $\alpha=\left(n_{i} \alpha_{i}\right)$ and all the $\alpha_{i}$ have the same order of magnitude as $\alpha$. If not, assume without loss of generality $\left|\alpha_{1}\right| \ll|\alpha|$ and let $\left(M_{\mathrm{g}, 0}-\alpha\right) \cup\left\{\alpha_{1}, \ldots, \alpha_{r}\right\}$ have one component a holed torus, with $\alpha_{1}, \ldots, \alpha_{r}$ in the interior of the holed torus. $\alpha_{2}, \ldots, \alpha_{r}$ can be found because all the components of $M_{g, 0}-\alpha$ are holed spheres (2.7) and $\alpha_{1}$ does not separate $M_{g, 0}(2.6)$. Then $\gamma_{\alpha}$ is obtained by 'skewing' $\left(n_{i}+4\right) \alpha_{i}$ as shown in figure 11, where $\alpha=\left(n_{i} \alpha_{i}\right)$.

Figure 11 gives an example when $r=2$. Identification of the circles marked $\sharp$ gives the one-holed torus. In general, suppose $\alpha_{i-1}, \alpha_{i+1}$ are the components adjacent to $\alpha_{i}$. Break across $\alpha_{i}$ at some point, and skew as shown in figure 12. By (2.8), you
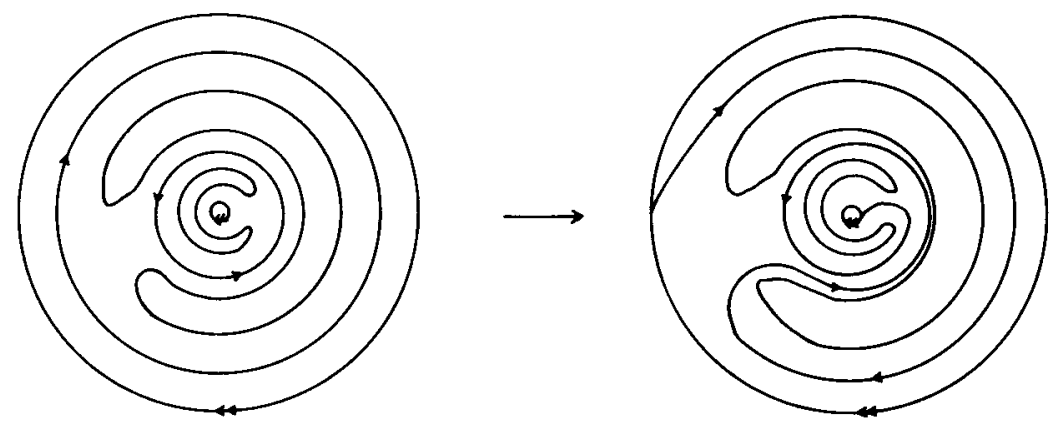

FIGURE 11 


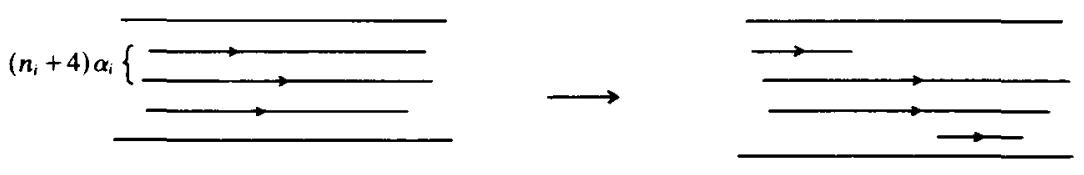

FIGURE 12

can continue the loose ends to meet $\alpha_{i-1}, \alpha_{i+1}$, forming a simple loop of length proportional to that of $\boldsymbol{\alpha}$. Then define

$$
F_{3, t+1}(\boldsymbol{\alpha})=\left\{n_{r+1} \alpha_{r+1}, \ldots, n_{s} \alpha_{s}\right\} \cup \gamma_{\boldsymbol{\alpha}} .
$$

$F_{3, t+1}$ is boundedly finite-to-one, and $F_{3, t+1}(\boldsymbol{\alpha})$ has the same order of magnitude as $\alpha$, in the same way as $F_{3}$ did for (2.2). $F_{4, t+1}(\boldsymbol{\alpha})$ is obtained by completing $F_{3, i+1}(\boldsymbol{\alpha})$ to a maximal loop, in the same way as $F_{4}$.

We now use proportions (2.2), (2.3), to construct certain subsets of $\operatorname{Diff}_{0}\left(M_{g, b}\right)$.

(2.9) Proposition. If $\boldsymbol{\alpha}=\left(\alpha_{1}, \ldots, \alpha_{3 g-3+b}\right)$ is a prime maximal loop with $\left|\alpha_{i}\right| /|\boldsymbol{\alpha}| \geq$ $B$, and $\phi \mathbf{e}=\alpha$, for some $\phi \in \operatorname{Diff}_{0}\left(M_{g, b}\right)$, then there exist $\varepsilon, L, M$ independent of $\boldsymbol{\alpha}$, and a Dehn twist $\psi$ round $\mathbf{e}$, such that

$$
P(\phi \circ \psi) \leq L|\boldsymbol{\alpha}| \text { on } \mathscr{S} \mathcal{M} \mathscr{F},
$$

and

$$
P(\phi \circ \psi) \geq M|\boldsymbol{\alpha}| \quad \text { on } B_{i}(\mathbf{e}, \varepsilon)=\{\mathscr{F} \in \mathscr{S} \mathcal{M} \mathscr{F}: i(\mathscr{F}, \mathbf{e}) \leq \varepsilon\} .
$$

Proof. Write $\mathbf{e}=\left(e_{1}, \ldots, e_{3 \mathbf{g}-3+b}\right)$. Fix another prime multiple loop $\mathbf{e}^{\prime}=$ $\left(e_{3 g-2+b}, \ldots, e_{6 g-6+2 b}\right)$ with none of the same isotopy components-which necessarily means that $\mathbf{e}, \mathbf{e}^{\prime}$ cut the surface into cells. For the first part, by (1.8), we only have to find a Dehn twist $\psi$ round $e$ for which

$$
\left|\phi \circ \psi^{2} \mathbf{e}^{\prime}\right| \leq L_{1}|\mathbf{\alpha}|=L_{1}\left|\phi^{2} \mathbf{e}\right|,
$$

to bound $P(\phi \circ \psi)$ on $\mathscr{S} \mathcal{M F}$. a cuts $M_{g, b}$ into 3-holed spheres. We merely have to choose $\psi$ so that the arcs of $\phi \circ \psi^{2} \mathbf{e}^{\prime}$ in each holed sphere of $M_{g, b}-\alpha$ do not wind round the boundary more than once (figure 13). In this way, $\left|\phi \circ \psi \mathbf{e}^{\prime}\right|$ will be bounded by $L_{1}|\boldsymbol{\alpha}|$, for some universal $L_{1}$.

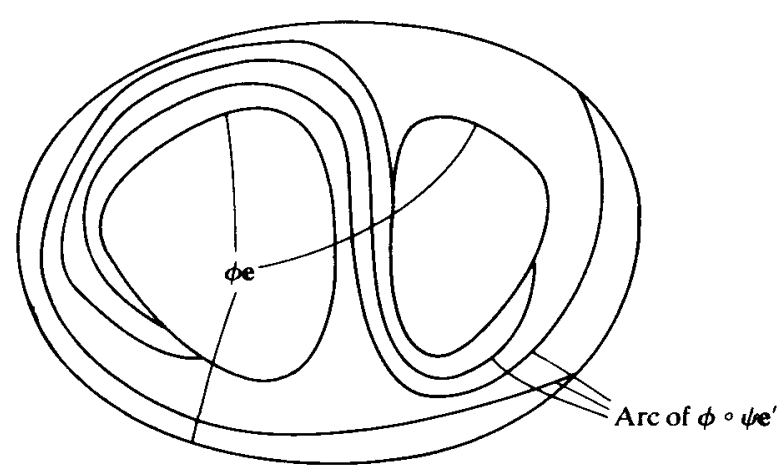

FIGURE 13 
For the second part, note that, for any $\varepsilon>0, B_{i}(\mathbf{e}, \varepsilon)$ will be contained in

$\left\{\mathscr{F}: d\left(\mathscr{F},\left(\lambda_{k} e_{k}\right)\right)<\eta\right.$ for some $\left.\left(\lambda_{k} e_{k}\right) \in \mathscr{P} \mathscr{M} \mathscr{F}\right\}$

for some $\eta>0$, for $d$ a fixed metric on $\mathscr{P} \mathscr{M F}$. By (1.6) $|\gamma \circ \psi \mathscr{F}|$ is boundedly proportional to

$$
\sum_{j=1}^{6 \mathrm{~g}-6+2 b} i\left(\phi \circ \psi \mathscr{F}, e_{j}\right)=\sum_{j=1}^{6 g-6+2 b} i\left(\mathscr{F},(\phi \circ \psi)^{-1} e_{j}\right) .
$$

So we may choose $\varepsilon$, using (1.11), so that for $\mathscr{F} \in B_{i}(\mathbf{e}, \varepsilon)$,

$$
|\phi \circ \psi \mathscr{F}| \geq M_{1} \inf _{\left(\lambda_{k} e_{k}\right) \in \mathscr{S} \mathcal{M} \mathscr{F}}\left(\left|\phi \circ \psi\left(\lambda_{k} e_{k}\right)\right|-a_{1} \sum_{j=1}^{6 g-6+2 b}\left|(\phi \circ \psi)^{-1} e_{i}\right|\right)
$$

for a suitable constant $a_{1}$.

The bound on $\left|\alpha_{i}\right|=\left|\phi \circ \psi e_{i}\right|$ gives, for $\mathscr{F} \in B_{i}(\mathbf{e}, \varepsilon)$,

$$
|\phi \circ \psi \mathscr{F}| \geq M_{2}|\boldsymbol{\alpha}|-a_{1} \sum_{j=1}^{6 g-6+2 b}\left|(\phi \circ \psi)^{-1} e_{j}\right|,
$$

where $a_{1}$ can be taken small compared with $M_{2}$, for suitable $\varepsilon$. By (1.8) we can bound

$$
\sum_{j=1}^{6 g-6+2 b}\left|(\phi \circ \psi)^{-1} e_{j}\right| \text { by } M_{3} \sup _{\mathscr{F} \in \mathscr{P} \mathcal{M F}}|(\phi \circ \psi) \mathscr{F}|,
$$

hence by $M_{4}|\boldsymbol{\alpha}|$. Thus for suitable $\varepsilon$ we obtain

$$
|\phi \circ \psi \mathscr{F}| \geq M|\boldsymbol{\alpha}| \quad \text { on } \quad B_{i}(\mathbf{e}, \varepsilon) \text {, some } M \text {. }
$$

We are now ready to prove:

(2.10) Proposition. Let e be any fixed prime maximal loop such that, for constants $A, B$, there exist $\geq B A^{(6 g-6+2 b)_{n}}$ loops $\phi \mathrm{e}$ of length $\geq A^{n},<A^{n+1}$, with all components $\varphi e_{i}$ of $\phi \mathbf{e}$ of length $\geq B A^{n}$ for $n \in F \subseteq \mathbb{N}, F$ infinite. (e exists by (2.2), since up to homeomorphism there are only finitely many ways a prime maximal loop can decompose $M_{\mathrm{g}, \mathrm{b}}$ into pairs of pants - all homeomorphisms of a pair of pants are isotopic to the identity.) Then, for any $\eta>0$, there exists a neighbourhood $U_{\eta}$ of $\left\{\left(\lambda_{i} e_{i}\right):\right.$ all $\left.\lambda_{i} \geq \eta\right\}$ and disjoint sets $\mathscr{B}_{n} \subseteq \operatorname{Diff}_{0}\left(M_{g, b}\right)$ with:

(i) $\sup _{\mathscr{F}_{\in} \in \mathscr{M} \mathscr{F}} P \phi(\mathscr{F})$ boundedly proportional to $A^{n}\left(\phi \in \mathscr{B}_{n}\right)$;

(ii) $P \phi$ boundedly proportional to $A^{n}$ on $U_{\eta} \cup\left\{e_{1}, \ldots, e_{3 g-3+b}\right\}$;

(iii) $\left\{\bar{\phi} U_{\eta}: \phi \in \mathscr{B}_{n}\right\}$ disjoint;

(iv) $\#\left(\mathscr{B}_{n}\right) \geq B A^{(6 \mathrm{~g}-6+2 b) n}$;

(v) (i)-(iv) imply measure $\left(\bigcup_{\phi \in \mathscr{B}_{n}} \bar{\phi} U_{\eta}\right) \geq C>0$, some $C$, for all $n$ (1.9).

Proof. This comes directly from (2.2), (2.9), except for disjointness which is obtained by suitable choice of $U_{\eta}$. But, clearly, we may assume the $\phi \mathbf{e}\left(\phi \in \mathscr{B}_{n}\right)$ are all distinct. Take

$$
U_{\eta}=\left\{\mathscr{F}: d\left(\mathscr{F},\left(\lambda_{i} e_{i}\right)\right)<\delta \text {, some }\left(\lambda_{i}\right) \text { with all } \lambda_{i} \geq \eta\right\},
$$

for some $\delta$ yet to be determined. Suppose $\bar{\psi} \mathscr{F} \in \bar{\phi} U_{\eta}, \phi, \psi \in \mathscr{B}_{n}, \mathscr{F} \in U_{\eta}$. Then

$$
\bar{\psi} \mathscr{F}=\bar{\phi} \mathscr{G},
$$

where $\mathscr{G} \in U_{\eta}$ and $i(\mathscr{G}, \mathbf{e}) \leq C_{1} \delta(1.10)$. Hence

$$
i(\bar{\psi} \mathscr{F}, \bar{\phi} \mathbf{e}) \leq C_{2} \delta / A^{2 n} .
$$


So $i\left(\mathscr{F}, \psi^{-1} \phi \mathbf{e}\right) \leq C_{3} \delta$, since $P \psi(\mathscr{F}) \leq C_{4} P \phi(\mathbf{e})=O\left(A^{n}\right)$. By $(1.10)$, if $d\left(\mathscr{F},\left(\lambda_{i} e_{i}\right)\right)<\delta$,

$$
i\left(\left(\lambda_{i} e_{i}\right), \psi^{-1} \phi \mathbf{e}\right) \leq C_{5} \delta\left|\psi^{-1} \phi \mathbf{e}\right| \text {. }
$$

Thus

$$
i\left(\mathbf{e}, \psi^{-1} \phi \mathbf{e}\right) \leq\left(C_{5} \delta / \eta\right)\left|\psi^{-1} \phi \mathbf{e}\right| .
$$

If $\varepsilon$ is as in (2.9), and $C_{5} \delta / \eta \leq \varepsilon$, then

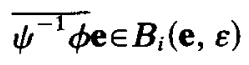

and

$$
P \psi\left(\psi^{-1} \phi \mathbf{e}\right)=O\left(A^{n}\right)
$$

so

$$
\left|\psi^{-1} \phi \mathbf{e}\right| \leq C_{6}
$$

Thus

$$
i\left(\mathbf{e}, \psi^{-1} \phi \mathbf{e}\right) \leq C_{6} C_{5} \delta / \eta
$$

that is,

$$
i(\psi \mathbf{e}, \phi \mathbf{e}) \leq C_{6} C_{5} \delta / \eta
$$

If $C_{6} X_{5} \delta / \eta<1$, this implies $i(\psi \mathbf{e}, \phi \mathbf{e})=0$, so

$$
\psi \mathbf{e}=\phi \mathbf{e} \text {, and } \phi=\psi \text {. }
$$

We shall need an analogue of $(2.10)$ for the interval exchange result. First a definition:

(2.11) Definition. Let $\mathscr{L}$ be a set of orientable foliations transverse to a prime multiple loop e as described just after (2.2). Let $\alpha \in \mathscr{L}$ be a maximal loop as an element of $\mathscr{L}$, that is, no isotopy components can be added to give another loop in $\mathscr{L}$. Let $\mathscr{L}_{\boldsymbol{\alpha}}$ denote the set of orientable foliations transverse to $\boldsymbol{\alpha}$, and having the same configuration as e in each holed sphere of $M_{\mathrm{g}, 0}-\alpha$ and as the $\mathscr{F}$ in $\mathscr{L}$ near e (we say this simply because, e being a loop, there are relations between the singularities of the associated foliation). Clearly,

$$
\phi \mathscr{L}_{\boldsymbol{\alpha}}=\mathscr{L}_{\phi \boldsymbol{\alpha}}
$$

if $\boldsymbol{\alpha}, \phi \alpha$ are both loops with all components transverse to e. So there are finitely many possibilities for $\mathscr{L}_{\alpha}$, up to conjugation. Also,

$$
\mathscr{L}=\left(\mathscr{L}_{\mathbf{\alpha}}\right)_{\mathbf{e}}
$$

so since clearly $\operatorname{dim} \mathscr{L}_{\alpha} \geq \operatorname{dim} \mathscr{L}, \mathscr{L}$ and $\mathscr{L}_{\alpha}$ have the same dimension.

(2.12) Proposition. Let $\mathscr{L}$ be a set of orientable foliations associated to e, as above. Then there exist $\mathbf{e}^{\prime}, \mathscr{L}^{\prime}$ with $\operatorname{dim} \mathscr{L}=\operatorname{dim} \mathscr{L}^{\prime}$, disjoint subsets $\mathscr{B}_{n} \subseteq \operatorname{Diff}_{0}\left(M_{g_{0} 0}\right)$, constants $A, B$, and (for $\eta>0$ ) a neighbourhood $U_{\eta}$ of $\left\{\left(\lambda_{i} e_{i}\right): \lambda_{i} \geq \eta\right\}$ in $\mathscr{L} \cap \mathscr{S M F}$ such that (i)-(v) of (2.10) hold, with (iv) replaced by:

(iv) $\#\left(\mathscr{B}_{n}\right) \geq B A^{(\operatorname{dim} \mathscr{L}) n} ;$ and

(vi) $\bar{\phi} U_{n} \subseteq \mathscr{L}^{\prime}\left(\phi \in \mathscr{B}_{n}\right)$.

Proof. Using (2.3), let $\mathscr{C}_{n}$ denote a set of prime maximal loops in $\mathscr{L}$ with

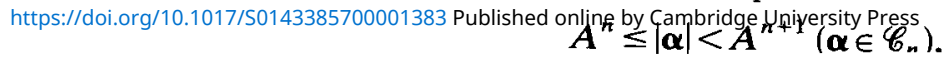


with lengths in bounded proportion and

$$
\#\left(\mathscr{C}_{n}\right) \geq B A^{n \operatorname{dim} \mathscr{L}} \text {. }
$$

As remarked above, there are only finitely many possibilities for $\mathscr{L}_{\boldsymbol{\alpha}}$ up to conjugation, so we may assume $\mathscr{L}_{\alpha}=\psi_{\alpha} \mathscr{L}_{\mathrm{f}}$, some fixed f. We may assume all $\alpha \in \mathscr{C}_{n}$ are in $X_{\delta, \mathrm{e}}(1.7)$ for some $\delta>0$ independent of $n$ (otherwise we would be unable to find $\geq B A^{n \operatorname{dim} \mathscr{L}}$ of them). In this case there is a fixed open

$$
U \subseteq \mathscr{L} \cap \mathscr{S M \mathscr { F }}, \quad U \supseteq\left\{\left(\lambda_{i} e_{i}\right): \text { all } \lambda_{i}>0\right\}
$$

which is contained in $\mathscr{L}_{\alpha}, \alpha \in U \mathscr{C}_{n}$ - because an $\alpha \in \mathscr{C}_{n}$ is boundedly transverse to an $\mathscr{F} \in U$, and the configuration of first hitting points of singular prongs of $\mathscr{F}$ on $\alpha \in \mathscr{C}_{n}$ is constant for $\mathscr{F} \in U$. Since $\mathscr{L}_{\mathrm{f}}$ is invariant under Dehn twists round $\mathbf{f}$, we may make

$$
\sup _{\mathscr{F} \in \mathscr{\mathscr { M }} \mathcal{M}}\left|\psi_{\mathbf{\alpha}} \mathscr{F}\right|
$$

boundedly proportional to $|\boldsymbol{\alpha}|$, by an analogous argument to that in (2.9). Then by (1.8),

$$
\sup _{\mathscr{F} \in \mathscr{S} \mathcal{M F}}\left|\psi_{\alpha}^{-1} \mathscr{F}\right|
$$

is also boundedly proportional to $|\boldsymbol{\alpha}|$. The lower bound on $\left|P \psi_{\alpha}^{-1}\right|$ on $U$ is just as in (2.9), since

$$
i\left(\psi_{\mathbf{\alpha}}^{-1} \mathbf{e}, \mathbf{f}\right)=i\left(\mathbf{e}, \psi_{\mathbf{\alpha}} \mathbf{f}\right)=i(\mathbf{e}, \mathbf{\alpha})=O(|\mathbf{\alpha}|)
$$

so

$$
\left|\psi_{\mathbf{\alpha}}^{-1} \mathbf{e}\right|=O(|\mathbf{\alpha}|)
$$

Take $\mathbf{e}^{\prime}=\mathbf{f}, \mathscr{L}^{\prime}=\mathscr{L}_{\mathbf{f}}$

$$
U \subseteq \mathscr{L}_{\alpha}=\psi_{\alpha} \mathscr{L}_{\mathbf{f}} \text { so } \psi_{\alpha}^{-1} U \subseteq \mathscr{L}_{\mathbf{f}} .
$$

We take $\mathscr{B}_{n}=\left\{\psi_{\alpha}^{-1}: \alpha \in \mathscr{C}_{n}\right\}$. We may assume the $\psi_{\alpha}^{-1}$ e are all distinct, because if $\psi_{\mathbf{\alpha}_{1}}^{-1} \mathbf{e}=\psi_{\mathbf{\alpha}_{2}}^{-1} \mathbf{e}$, then $\psi_{\mathbf{\alpha}_{2}} \circ \psi_{\mathbf{\alpha}_{1}}^{-1}$ is a Dehn twist round e. There are only finitely many such Dehn twists $T$ with

$$
\sup \{|T \mathscr{F}|: T \mathscr{F} /|T \mathscr{F}| \in U\} \leq M
$$

(for any constant $M$ ). Since

$$
\psi_{\mathbf{a}_{1}}^{-1}=\psi_{\mathbf{\alpha}_{2}}^{-1} \circ\left(\psi_{\mathbf{\alpha}_{2}} \circ \psi_{\mathbf{\alpha}_{1}}^{-1}\right)
$$

and $\left|\psi_{\alpha_{1}}^{-1}\right|,\left|\psi_{\alpha_{2}}^{-1}\right|$ are boundedly proportional on $U$, the map $\alpha \mapsto \psi_{\alpha}^{-1}$ is at most boundedly finite-to-one. The argument making $\left\{\psi_{\boldsymbol{\alpha}}^{-1} U_{\eta}: \alpha \in \mathscr{B}_{\eta}\right\}$ disjoint for suitable $U_{\eta}$ is then exactly as in (2.10).

3. Proofs of theorems 1, 2 and 3

We claim that, in order to prove theorem 1 , it suffices to prove the following:

(3.1) Proposition. There exists open $U \subseteq \mathscr{P} M \mathscr{F}$, and subsets $\mathscr{B}_{n}(n \in F)$ of $\operatorname{Diff}_{0}\left(M_{g, b}\right)$ with $F \subseteq \mathbb{N}$ infinite, $\left\{\bar{\phi} U: \phi \in \mathscr{B}_{n}\right\}$ all disjoint, $d \phi_{*} \lambda / d \lambda$ proportional on $U$ to $A^{-n(6 g-6+2 b)}$ (some $\left.A\right),\left(\bigcup_{\phi \in B_{n}} \bar{\phi} U\right) \geq C>0$ and such that the following holds. 
For each $\delta>0$ there exists $N=N(\delta)$ such that, for $n \geqslant N$, there exists $\mathscr{B}_{n, \delta}$ with

$$
\lambda\left(\bigcup_{\phi \in \mathscr{P}_{n}-\mathscr{B}_{n . \delta}} \bar{\phi} U\right)<\delta
$$

and there exists, for each $\bar{\phi} U\left(\phi \in \mathscr{B}_{n, \delta}\right)$ a d-ball of radius $\delta$ such that, if $U_{\phi}$ denotes the intersection of $\bar{\phi} U$ with this d-ball, then $\lambda\left(U_{\phi}\right) / \lambda(\bar{\phi} U)>1-\delta$.

Note. $U, F$, and the $\mathscr{B}_{n}$ will be subsets of the $U, F, \mathscr{B}_{n}$ constructed in proposition (2.10). ( $\eta$ can be taken just so that $U_{\eta}$ contains the projection of e on $\mathscr{S} \mathcal{M F}$, for the measured foliations result. It is for the interval exchange result that we need to be able to vary $\eta$.) $F, U, \mathscr{B}_{n}$ will be made to have properties additional to those listed in (2.10). $d$ denotes one of the natural Lipschitz-equivalent metrics on $\mathscr{P} \mathcal{M} \mathscr{F}$. It will be convenient to choose $d$ so that $d$-balls have piecewise smooth boundary ( $d$ given piecewise by Euclidean norm metrics, for example). It will also be convenient to choose $U$ with piecewise smooth boundary.

Proof of sufficiency for theorem 1 . Choose $\left\{\varepsilon_{n}\right\}$ with $\varepsilon_{n} \rightarrow 0$. Inductively construct $\delta_{n}<\frac{1}{2} \varepsilon_{n}, r_{n} \geq N\left(\delta_{n}\right), r_{n} \in \mathscr{F}$, and $\mathscr{B}_{r_{n}}^{\prime} \subseteq \mathscr{B}_{r_{n}, \delta_{n}}$ with

$$
\lambda\left(\underset{\phi \in \mathscr{B}_{\boldsymbol{F}_{n}}-\mathscr{B}_{r_{n}}^{\prime}}{\bigcup_{\boldsymbol{\phi}}} \bar{U}\right)<\varepsilon_{n},
$$

and such that, for any $U_{\phi}, U_{\psi}, \phi \in \mathscr{B}_{r_{n}}, \psi \in \mathscr{B}_{r_{m}}, m<n$, either

$$
U_{\phi} \subseteq U_{\psi} \text { or } U_{\phi} \cap U_{\psi}=\varnothing \text {. }
$$

This is possible. For, inductively, we can choose $\delta_{n+1}$ so that the $3 \delta_{n+1}$-neighbourhood of

$$
\bigcup\left\{\text { Boundary } U_{\psi}: \psi \in \bigcup_{m \leq n} \mathscr{B}_{r_{m}}^{\prime}\right\}=X_{n}
$$

has measure $<\varepsilon_{n+1} / D$. We can then cover $X_{n}$ by $\delta_{n+1}$-balls so that the sum of the measures of $3 \delta_{n+1}$-balls with the same centres is $<\frac{1}{4} \varepsilon_{n+1}$. ( $D$ depends only on the choice of metric.) Then take $\mathscr{B}_{r_{n}}^{\prime} \subseteq \mathscr{B}_{r_{n}, \delta_{n}}$ to be those $\phi$ for which $U_{\phi}$ does not lie in one of the $3 \delta_{n+1}$-balls. Let

$$
E=\bigcap_{N=1}^{\infty} \bigcup_{\substack{n=N \\ n \in F}}^{\infty} \bigcup_{\phi \in \mathscr{P}_{r_{n}^{\prime}}} U_{\phi} .
$$

Then $\lambda(E) \geq C^{\prime}>0$. To prove theorem 1, it suffices to show that the orbit of $E$ under $\operatorname{Diff}_{0}\left(M_{g, b}\right)$ has full measure, and that $\operatorname{Diff}_{0}\left(M_{g, b}\right)$ acts ergodically on this orbit - the same idea as in [6]. But for any positive measure $E_{1} \subseteq E$, we can find arbitrarily close coverings by disjoint $U_{\phi}, \phi \in \bigcup_{n \geq N} \mathscr{B}_{r_{n}}^{\prime}$ (because any covering has a refinement of disjoint $\left.U_{\phi}\right)$. So for any $\varepsilon>0$, and any $N$, we can find $U_{\phi}$ with

$$
\lambda\left(E_{1} \cap U_{\phi}\right) / \lambda\left(U_{\phi}\right)>1-\varepsilon, \quad \phi \in \mathscr{B}_{r_{n}}^{\prime}, n \geq N .
$$

Then

$$
\lambda\left(\bar{\phi}^{-1}\left(E_{1}\right) \cap U\right) / \lambda(U) \geq 1-B \varepsilon,
$$

for some constant $B$, by the boundedness of the variation of $P \phi$ on $U$, and closeness in measure of $U_{\phi}$ to $\bar{\phi} U$. So $U$ is almost all contained in the orbit of $E_{1}$. By 
minimality of the action of $\operatorname{Diff}_{0}\left(M_{g, b}\right)$ on $\mathscr{P} \mathscr{M} \mathscr{F}[2], \mathscr{P} \mathscr{M} \mathscr{F}$ is almost all contained, which is what we need.

Theorem 2 will follow from the method of proof of (3.1). In fact, we shall prove that all $\mathscr{F}$ in

$$
\bigcap_{N=1}^{\infty} \bigcup_{\substack{n \geq N \\ n \in F}} \bigcup_{\phi \in \mathscr{B}_{n}} \bar{\phi} U
$$

are uniquely ergodic - and then use of theorem 1 to deduce that the set of uniquely ergodic foliations has full measure.

(3.2) The metric on $\mathscr{P} \mathcal{M} \mathscr{F}$. One of the main ingredients in the proof of (3.1) is to reinterpret closeness of foliations in the $d$-metric, for $d$ one of the natural Lipschitzequivalent metrics. Suppose $\mathscr{F}, \mathscr{F}$ ' are transverse to a fixed prime maximal loop e. (3.2.1), (3.2.2) give sufficient conditions for $\mathscr{F}, \mathscr{F}$ ' to be close in the $d$-metric.

(3.2.1). For each $e_{i}$, the set of different homotopy classes occurring for arcs of $\mathscr{F}$-leaves with endpoints on e, and crossing just $e_{i}$ in between the endpoints, is the same as the set for $\mathscr{F}^{\prime}$. For example, for the surface $M_{2,0}$, and $\mathbf{e}$ as shown, the set of homotopy classes for $e_{2}$ might be as shown in figure 14. We allow free homotopy

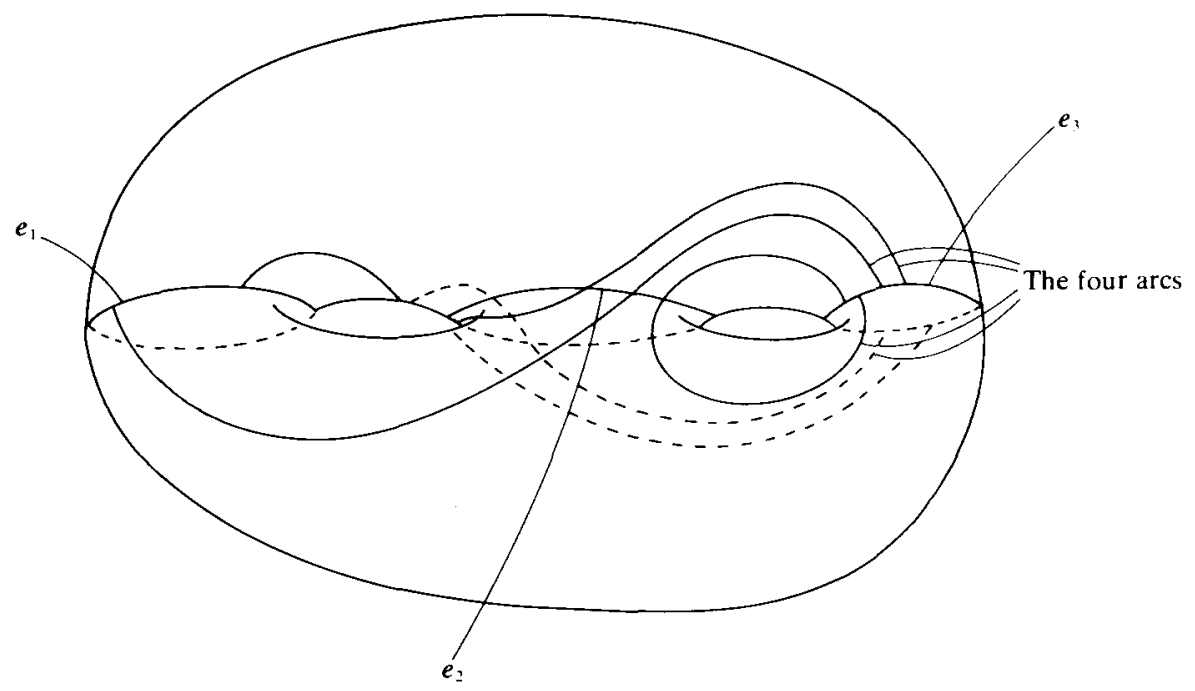

FIGURE 14

of the endpoints of the arcs along e. Note that the arcs in the set are always non-intersecting, up to homotopy. Now, the different homotopy classes determine a partition of $e_{i}$, for each $i$. The partition intervals are intervals between first hitting points on $e_{i}$ of singular prongs of $\mathscr{F}_{\text {. Let }} f_{1}, \ldots, f_{k}$ be an enumeration of the characteristic functions of these partition intervals, for all $e_{i}$. Then $\mu_{\mathscr{F}}\left(f_{1}\right), \ldots, \mu_{\mathscr{F}}\left(f_{k}\right)$ are determined by the $m_{j}(\mathscr{F}), p_{i}(\mathscr{F})$ of $(1.1)$. Conversely, the set of homotopy classes plus the $\mu_{\mathscr{F}}\left(f_{i}\right)$ determine the $m_{j}(\mathscr{F}), p_{j}(\mathscr{F})$. 
The second condition for $\mathscr{F}, \mathscr{F}$ ' to be close is:

(3.2.2) Assuming (3.2.1) holds, $\left|\mu_{\mathscr{F}}\left(f_{i}\right)-\mu_{\mathscr{F}}\left(f_{i}\right)\right|$ is small for each $i$, with the notation above.

(3.3) The restrictions of $F, \mathscr{B}_{n}$. In addition to the restrictions of (2.10) on $U$ open in $\mathscr{P} \mathcal{M} \mathscr{F}, \mathscr{B}_{n} \subseteq \operatorname{Diff}_{0}\left(M_{g, b}\right)(n \in F), F \subseteq N$ infinite, we can assume:

(3.3.1) $\phi \mathbf{e}\left(\phi \in \mathscr{B}_{n}\right)$ all lie in $X_{\mathbf{e}, \nu}$, for some fixed $\nu>0\left(X_{\mathbf{e}, \nu}\right.$ as in (1.7)).

This is because, if $\nu$ is sufficiently small,

$$
\#\left\{\boldsymbol{\alpha}: \boldsymbol{\alpha} \text { is a multiple loop, } \alpha \notin X_{\mathrm{e}, \nu}, A^{n} \leq|\boldsymbol{\alpha}|<A^{n+1}\right\}<\varepsilon A^{n},
$$

given $\varepsilon$ (because, associating $\alpha$ to the $\left(m_{i}, p_{i}\right)$ coordinates of (1.1) taken with respect to the loop e, $\alpha \notin X_{\mathbf{e}, \nu}$ means at least one $m_{i} / p_{i}$ is small). But then the sums of measures of $\bar{\phi} U, \phi \mathbf{e} \notin X_{\mathbf{e}, \nu}$ will be much less than the $C$ for which $\sum_{\phi \in \mathscr{B}_{n}} \lambda(\bar{\phi} U) \geq C$. Reducing $C$ slightly, and reducing the size of the $\mathscr{B}_{n}$ slightly, we have the result. (3.3.2) Regarding $\phi \mathbf{e}$ as a measured foliation, first hitting points of singular prongs of $\phi \mathbf{e}$ on $e_{i}$ are all distinct, for each $i$, and in each pair of pants defined by $\mathbf{e}$, singularities are 3-pronged, for all $\phi \in \bigcup_{n} \mathscr{B}_{n}$.

This can be done because the set of foliations for which singular prongs coincide on $e_{i}$, as shown in figure 15 , or for which singularities have more than three prongs, is of proper codimension in the set of measured foliations.

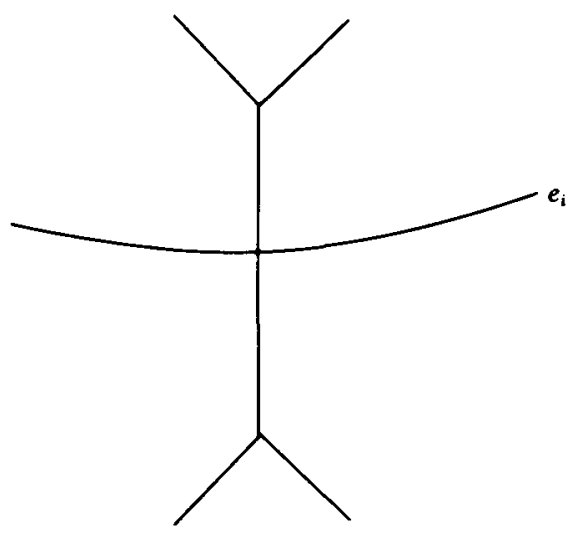

FIGURE 15

(3.3.3) Regarding $\phi \mathbf{e}$ as a measured foliation, for each $e_{i}$, the set of homotopy arcs associated to $e_{i}$ and $\phi \mathbf{e}$, as in (3.2.1), is the same for all $\phi \in \cup_{n} \mathscr{B}_{n}$.

This can be done because, once we know all $\phi \mathbf{e}$ are in $X_{\mathbf{e}, \nu}$ by (3.3.1), there is only a finite number of possible sets of homotopy arcs. Reduce $F$, and the constant $C$ if necessary.

Note. If $f_{1}, \ldots, f_{k}$ is an enumeration of the characteristic functions described in (3.2.1) for one and hence (by (3.3.3)) for all $\phi \mathbf{e}$, then $\mu_{\phi e_{i}}\left(f_{i}\right)$ are defined as well as $\mu_{\phi \mathbf{e}}\left(f_{i}\right)$-although some of the $\mu_{\phi e_{i}}\left(f_{j}\right)$ may be 0 . 
We shall use the following form of the:

(3.4) Birkhoff ergodic theorem. Let $(X, T, \mu)$ be a probability measure space, $T$ measure-preserving invertible, $f \in L^{\infty}(\mu)$. Let

$$
S_{n, m}(f)=\frac{1}{n-m} \sum_{i=m}^{n-1} f \circ T^{i}, \quad n>m
$$

Then for any $a>0$,

$$
A_{N, a}=\int\left[\sup _{\substack{n \geq N \\ n-m \geq a n}} S_{n, m}(f)-\inf _{\substack{n \geq N \\ n-m \geq a n}} S_{n, m}(f)\right] d \mu \rightarrow 0 \text { as } N \rightarrow \infty .
$$

Also, $\mu(f)$ is sandwiched between $\int$ inf and $\int$ sup.

Proof.

$$
S_{n, m}(f)=\frac{n}{n-m} S_{n, 0}(f)-\frac{m}{n-m} S_{m, 0}(f)
$$

So

$$
\begin{aligned}
A_{N, a} \leq & \left.\frac{1}{a} \int \sup _{n \geq N} S_{n, 0}(f)-\inf _{n \geq N} S_{n, 0}(f)\right) d \mu \\
& +\frac{1}{a} \int\left(\sup _{n \geq m \geq N} \frac{m}{n} S_{m, 0}(f)-\inf _{n \geq m \geq N} \frac{m}{n} S_{m, 0}(f)\right) d \mu .
\end{aligned}
$$

Both terms tend to 0 . The second term tends to 0 because, when $m$ is small, $m / n$ is small, and

$$
\left|S_{m, 0}(f)\right| \leq\|f\|_{\infty}
$$

When $m$ is larger, the usual form of the Birkhoff theorem makes the term tend to 0 .

We now claim that (3.1) (hence theorem 1) and theorem 2 are implied by:

(3.5) Proposition. For a suitable choice of $U$, all $\mathscr{F}$ in $\bar{\phi} U$ are 'close' enough to $\phi \mathbf{e}$ for (3.2.1) to be satisfied with $\mathscr{F}^{\prime}=\phi \mathbf{e}$. That is, all $\mathscr{F}$ in $\bar{\phi} U$ give the same sets of homotopy arcs as $\phi \mathbf{e}$ (hence the same as all $\psi \mathbf{e}, \psi \in \bigcup \mathscr{B}_{n}$ by (3.3.3)). Further, let $f_{1}, \ldots, f_{k}$ be an enumeration of the characteristic functions as in (3.2.1). Let $\mathrm{e} \times\{-1,1\}$ denote the inverse image of $\mathbf{e}$ in the orientable cover of $\mathscr{F}$. (If $\mathscr{F}$ is already orientable, take two copies.) Let $T_{\mathscr{F}}$ denote the first return map of the cover of $\mathscr{F}$ to $\mathbf{e} \times\{-1,1\}$. Let

$$
S_{n, m, \mathscr{F}}\left(f_{k}\right)=\frac{1}{n-m} \sum_{i=m}^{n-1} f_{k} \circ T_{\mathscr{F} .}^{i}
$$

Then, for constants $D_{1}, D_{2}$,

$$
\inf _{\substack{n \geq D_{1} A^{p} \\ n-m \geq D_{2^{n}}}} S_{n, m, \mathscr{F}}\left(f_{k}\right) \leq \mu_{\phi e_{i}}\left(f_{k}\right) \leq \sup _{\substack{n \geq D_{1} A^{p} \\ n-m \geq D_{2} n}} S_{n, m, \mathscr{F}}\left(f_{k}\right)
$$

for all $k, j$, where $\phi \mathbf{e} \in \mathscr{B}_{p}$. Here, we normalize the measure $\mu_{\phi e_{j}}$ to let $\mathbf{e} \times\{-1,1\}$ have measure 1. 
Proof of sufficiency of (3.5) for (3.1). Let $\mu_{\mathscr{F}}$ be the transverse measure of $\mathscr{F}$, which can be regarded as a $T_{\mathscr{F}}$-invariant measure on $\mathbf{e} \times\{-1,1\}$. Write $A_{N}\left(\mathscr{F}_{,}, k\right)$ for the quantity $A_{N, a}$ occurring in (3.4), with $\mu=\mu_{\mathscr{F}} f=f_{k}$ and $a=D_{2}$. Then, by (3.4),

$$
A_{N}(\mathscr{F}, k) \rightarrow 0 \text { as } N \rightarrow \infty
$$

for $k$, and all $\mathscr{F} \in \bigcup_{n \in F} \bigcup_{\mathscr{\phi} \in \mathscr{B}_{n}} \bar{\phi} U . A_{N}(\mathscr{F}, k)$ is a measurable function of $\mathscr{F}$. So, given $\delta$, we can choose $N=N(\delta)$ so that

$$
A_{N}(\mathscr{F}, k)<\delta
$$

for all $k$, and all $\mathscr{F} \in Y_{N}$, where

$$
\lambda\left(\bigcup_{n \in F} \bigcup_{\phi \in \mathscr{P}_{n}} \bar{\phi} U \backslash Y_{N}\right)<\delta^{2}
$$

Then put

$$
\mathscr{B}_{p, \delta}=\left\{\phi \in \mathscr{B}_{p}: \frac{\lambda\left(Y_{N} \cap \bar{\phi} U\right)}{\lambda(\bar{\phi} U)}>1-\delta\right\}
$$

for $D_{1} A^{p} \geq N$. Then

$$
\lambda\left(\bigcup_{\phi \in \mathscr{B}_{p}-\mathscr{B}_{p . \delta}} \tilde{\phi} U\right)<\delta
$$

By the last part of (3.4), and (3.5), we have

$$
\left|\mu_{\mathscr{F}}\left(f_{k}\right)-\mu_{\phi e_{i}}\left(f_{k}\right)\right|<\delta
$$

for all $k, j$, and for all $\mathscr{F} \in \bar{\phi} U \cap Y_{N}, \phi \in \mathscr{B}_{p}, D_{1} A^{p} \geq N$. This gives (3.1) due to the interpretation of the metric in (3.2).

Proof of sufficiency of (3.5) for theorem 2. Take any $\mathscr{F}$ in $\bigcap_{N=1}^{\infty} \bigcup_{n \geq N, n \in F} \bar{\phi} U$. Let $\nu_{\mathscr{F}}$ be any transverse $\mathscr{F}$-invariant measure, regarded as a measure on $\mathbf{e} \times\{-1,1\}$ invariant under $T_{\mathscr{F} \text {. If }} A_{N}(\mathscr{F}, k)$ as above is defined with $\nu_{\mathscr{F}}$ in place of $\mu_{\mathscr{F}}$, we again have

$$
\lim _{N \rightarrow \infty} A_{N}(\mathscr{F}, k)=0 \quad \text { for all } k \text {. }
$$

Using the last part of (3.4) and (3.5), as in the proof of sufficiency for (3.1), we deduce:

$$
\lim _{\mathscr{F} \in \bar{\phi} U, \phi \in \mathscr{P}_{p}, p \rightarrow \infty}\left|\nu_{\mathscr{F}}\left(f_{k}\right)-\mu_{\phi e_{i}}\left(f_{k}\right)\right|=0 \quad \text { for all } j, k .
$$

Thus $(\S 1$, before $(1.12)) \nu_{F}$ is unique, so $\mathscr{F}$ is uniquely ergodic.

(3.5) follows from:

(3.6) Proposition. We can choose $U$ so that for a constant $L$, for all $p$, for each $\mathscr{F} \in \bar{\phi} U\left(\phi \in \mathscr{B}_{p}\right)$, each segment of a leaf of $\mathscr{F}$ which passes through $\geq L A^{p}$ of the pairs of pants defined by e contains, for each $j$, a subsegment with both ends lying on an $e_{i}$, such that the subsegment gives a loop isotopic to twice $\phi e_{j}$ when joined along a segment of $e_{i}$ which twists round $e_{i}$ less than once. 
Proof of (3.5) from (3.6). (3.6) shows that the set of homotopy arcs associated to $\phi \mathbf{e}$, regarding $\phi \mathbf{e}$ as a measured foliation (3.2.1), is contained in the set associated to $\mathscr{F}$, for each $\mathscr{F}$ in $\bar{\phi} U$. But (3.3.2) says that the set of arcs for $\phi \mathbf{e}$ cannot be enlarged - so $\mathscr{F} \in \bar{\phi} U$ and $\ddot{\phi}$ e are close in the sense of (3.2.1). (3.6) implies the second part of (3.5) because $\phi e_{j}$ passes through between $K_{1} A^{p}$ and $K_{2} A^{p}$ pairs of pants durings its circuit, by (3.3.1). So for each $x \in \mathbf{e} \times\{-1,1\}$, there exist $n, m$, $0<m<n \leq L A^{p}, n-m \geq K_{1} A^{p}$, such that

$$
S_{n, m, \mathscr{F}}\left(f_{k}\right)(x)=\mu_{\phi e_{j}}\left(f_{k}\right) .
$$

(Of course, $n, m$ vary with $x$.)

For (3.6) we need:

(3.7) LEMMA. Under the assumption that all $\phi \mathbf{e}$ lie in $X_{\mathbf{e}, \nu}(3.3 .1)$, all $\phi^{-1} \mathbf{e}$ lie in $X_{\mathrm{e}, \nu_{1}}$, some $\nu_{1}>0$.

Proof. Since $\phi \mathbf{e} \in X_{\mathbf{e}, \nu}$, and the $\left|\phi e_{k}\right|$ are in bounded proportion (2.10), there exists $H_{1}$ such that

$$
|\phi \mathbf{e}| \leq H_{1} i\left(\phi e_{k}, \mathbf{e}\right) \quad \text { for all } k, \phi \text {. }
$$

Since

$$
\begin{gathered}
|\phi \mathbf{e}| \geq H_{2} \sup _{\mathscr{F} \in \mathscr{P M} \mathscr{F}}|\phi \mathscr{F}| \quad \text { by }(2.10) \text { and }(1.8), \\
\left|\phi^{-1} \mathbf{e}\right| \leq H_{3} i\left(\phi e_{k}, \mathbf{e}\right)=H_{3} i\left(\phi^{-1} \mathbf{e}, e_{k}\right) .
\end{gathered}
$$

Proof of (3.6). Recall that, so far, the only restriction on $U$ is that it be contained in a sufficiently small neighbourhood of $\left\{\left(\lambda_{i} e_{i}\right)\right.$ : all $\left.\lambda_{i} \geq \eta\right\}$, for any fixed $\eta>0$. By (3.7), the integral twist of $\phi^{-1}$ e round each $e_{j}\left(\phi \in \mathscr{B}_{n}\right)$ is $\leq G$, some constant $G$. Restrict the open set $U$ to a set of foliations for which all leaves crossing $e_{j}$ twist round $e_{j} \geq G+3$ times for all $j$ - take $U$ as a sufficiently small neighbourhood of e, for example. Then, for all leaves crossing $e_{j}$, we can find a segment of the leaf for which both endpoints lie on a segment of $\phi^{-1} e_{i}$, for some $i$, and such that the loop formed by the union of the leaf-segment and the $\phi^{-1} e_{i}$-segment is isotopic to twice $e_{j}$ (figure 16).

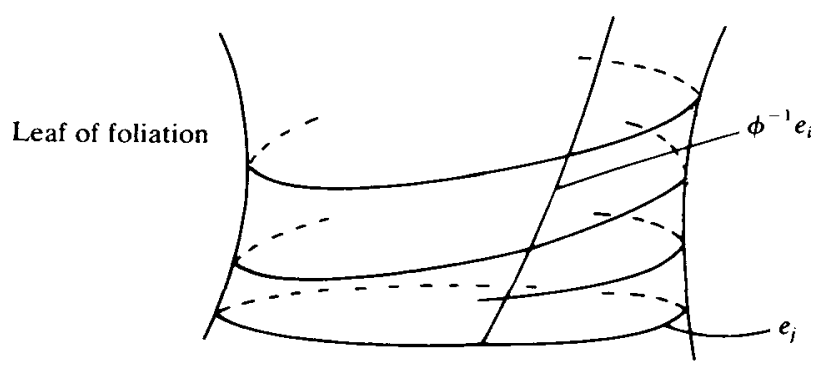

FIGURE 16 
Restrict $U$ further to a neighbourhood of a minimal foliation $\mathscr{F}_{0}$, where all leaves of $\mathscr{F}_{0}$ cross all $e_{j}$ before passing through $\boldsymbol{M}$ of the pairs of pants defined by $\mathbf{e}$, for some $M$. This is possible by (1.13), and we can clearly make the same property hold for all $\mathscr{F} \in U$, by the choice of $U$. So, by (3.7), all leaves cross $e_{j}$, hence twist round $e_{j} \geq G+3$ times, before crossing $\phi^{-1}$ e $L|\phi \mathbf{e}|$ times, for some constant $L$. Acting by $\phi$, we see that this implies that all leaves of $\mathscr{F} \in \bar{\phi} U$ wind twice around $\phi e_{j}$ before crossing e $L|\phi \mathbf{e}|$ times, as required.

\section{Modifications for theorem 3}

As in Masur's paper, the problem of showing that almost all interval exchanges are uniquely ergodic is equivalent to another problem about orientable foliations. In fact, we need:

(3.8) THEOREM. Let $\mathscr{L}$ be a set of orientable foliations with configuration fixed with respect to a prime loop $\mathbf{e}$, as described at the beginning of $\S 2$. Then almost all foliations in $\mathscr{L}$ are uniquely ergodic.

We should explain why we need this. We first claim that a minimal oriented foliation with no leaves connecting different singularities lies in an $\mathscr{L}$. This is exactly the same argument as in [4] theorem II.1 (but simpler - for minimal foliations we do not need lemma II.1). Conversely, non-minimal foliations in $\mathscr{L}$ form a countable union of lower dimensional subvarieties. For, by the same argument as in $\S 2$ (2.6), no foliation in $\mathscr{L}$ contains a separating cycle of leaves - but a non-separating cycle can always be lost inside $\mathscr{L}$, by a small Dehn twist round a loop not intersecting singularities. (We only give this sketch, as we do not really need to know the fact for the proof of theorem 3.)

We can now claim that a suspension of an interval exchange $f_{\lambda, \sigma}$ (with the notation of the introduction) lies in an $\mathscr{L}$, since a residual full measure set of $f_{\lambda, \sigma}$ consists of minimal interval exchanges with the orbits of discontinuities infinite and distinct [3]. The suspension (for definition see [1] or [4], for example) of such an $f_{\lambda, \sigma}$ is thus minimal with no liaisons between singularities - and at such an $f_{\lambda, \sigma}$, the map from the interval exchanges to their suspensions in an $\mathscr{L}$ is clearly open - which is why it suffices to prove (3.8).

Proof of (3.8). Let e be the prime multiple loop occurring in the definition of $\mathscr{L}$ given in $\S 2$. Since $\mathscr{L}$ is invariant under Dehn twists round e, it suffices to prove unique ergodicity almost everywhere on a neighbourhood $U_{\mathscr{G}_{0}}$ of every minimal foliation $\mathscr{F}_{0}$ in $\mathscr{L}$ sufficiently close to $\left\{\left(\lambda_{i} e_{i}\right) \in \mathscr{P} \mathcal{M} \mathscr{F}: \lambda_{i} \geq 0\right\}$. This is done by applying (3.1) to $U_{\mathscr{S}_{0}}$, and $\left\{\mathscr{B}_{n}\right\}$ of $(2.11)$ in place of $U_{1}$, and $\left\{\mathscr{B}_{n}\right\}$ of (2.10). Note that the Radon-Nikodym derivative of $\bar{\phi}: U_{\mathscr{F}_{0}} \rightarrow \bar{\phi} U_{\mathscr{F}_{0}}$ is boundedly proportional to $1 /|P \phi \mathscr{F}|^{s}, s=\operatorname{dim} \mathscr{L}$. All the $\bar{\phi} U_{\mathscr{F}_{0}}$ lie in a fixed $s$-dimensional space $\mathscr{L}^{\prime}$ (as in (2.11)). Apart from this, (3.1) needs no modification.

The author gratefully acknowledges the financial support of the Stiftung Volkswagenwerk for a visit to the IHES, during which this paper was written. 


\section{REFERENCES}

[1] P. Arnoux. Echanges d'intervalles et flots sur les surfaces. Monographie No. 29 de l'Enseignement Mathématique, pp. 5-38. Université de Genève.

[2] A. Fathi, F. Laudenbach \& V. Poenaru et al. Travaux de Thurston sur les surfaces. Astérisque 66-67 (1979).

[3] M. Keane. Interval exchange transformations. Math. Z. 141 (1975), 25-31.

[4] G. Levitt. Pantalons et feuilletages des surfaces. Topology 21 (1982), 9-34.

[5] H. Masur. Interval exchange transformations and measured foliations. Ann. Math. 115 (1982), 169-200.

[6] D. Sullivan. The density at infinity of a discrete group of hyperbolic motions. IHES Publ. Math. 50 (1979), 171-202.

[7] W. Veech. Gauss measures for transformations on the space of interval exchange maps. Ann. Math. 115 (1982), 201-242. 